\title{
Mutation of kri1/ causes definitive hematopoiesis failure via PERK-dependent excessive autophagy induction
}

\author{
Xiao-E Jia ${ }^{1,4,}{ }^{*}$, Ke $\mathrm{Ma}^{1, *}$, Tao $\mathrm{Xu}^{1, *}$, Lei Gao ${ }^{1}$, Shuang $\mathrm{Wu}^{1}$, Cong Fu${ }^{1}$, Wenjuan Zhang ${ }^{1}$, Zhizhang Wang ${ }^{1}$, \\ Kaiyu Liu ${ }^{6}$, Mei Dong ${ }^{1}$, Changbin Jing ${ }^{1}$, Chunguang Ren ${ }^{1}$, Zhiwei Dong ${ }^{1}$, Yi Chen ${ }^{2}$, Yi Jin ${ }^{2}$, Qiuhua Huang ${ }^{2}$, \\ Xing Chang ${ }^{1,9}$, Min Deng ${ }^{1}, \mathrm{Li} \mathrm{Li}^{8}$, Lingfei Luo ${ }^{8}$, Jun Zhu, ${ }^{7,9}$, Yongjun Dang ${ }^{6}$, Hung-Chun Chang, Leonard I Zon ${ }^{3}$, \\ Yi Zhou ${ }^{3}$, Saijuan Chen ${ }^{2,9}$, Weijun Pan ${ }^{1,9}$ \\ ${ }^{I}$ Key Laboratory of Stem Cell Biology, Institute of Health Sciences, Shanghai Institutes for Biological Sciences, Chinese Academy \\ of Sciences \& Shanghai Jiao Tong University School of Medicine, Shanghai, China; ${ }^{2}$ State Key Laboratory for Medical Genom- \\ ics, Shanghai Institute of Hematology, RuiJin Hospital, Shanghai Jiao Tong University School of Medicine, Shanghai, China; \\ ${ }^{3}$ Stem Cell Program, Hematology/Oncology Program at Children's Hospital Boston, Harvard Medical School, Boston, MA 02114, \\ USA; ${ }^{4}$ Biomedical Research Center, Baotou Medical College, Baotou, Inner Mongolia Autonomous Region, China; ${ }^{5}$ Institute of \\ Neuroscience, Shanghai Institutes for Biological Sciences, Chinese Academy of Sciences, Shanghai, China; ${ }^{6}$ Key Laboratory of \\ Metabolism and Molecular Medicine, Ministry of Education, Department of Biochemistry and Molecular Biology, School of Basic \\ Medical Sciences, Fudan University, Shanghai, China $;{ }^{7}$ CNRS LIA, Shanghai Institute of Hematology, RuiJin Hospital, 197 RuiJin \\ Road II, Shanghai, China; ${ }^{8}$ Key Laboratory of Freshwater Fish Reproduction and Development, Ministry of Education, Laborato- \\ ry of Molecular Developmental Biology, School of Life Sciences, Southwest University, Beibei, Chongqing, China; ${ }^{9}$ Collaborative \\ Innovation Center of Systems Biomedicine, Shanghai Jiao Tong University School of Medicine, Shanghai, China
}

Dysregulation of ribosome biogenesis causes human diseases, such as Diamond-Blackfan anemia, del (5q-) syndrome and bone marrow failure. However, the mechanisms of blood disorders in these diseases remain elusive. Through genetic mapping, molecular cloning and mechanism characterization of the zebrafish mutant cas002, we reveal a novel connection between ribosomal dysfunction and excessive autophagy in the regulation of hematopoietic stem/progenitor cells (HSPCs). cas002 carries a recessive lethal mutation in krill gene that encodes an essential component of rRNA small subunit processome. We show that Krill is required for normal ribosome biogenesis, expansion of definitive HSPCs and subsequent lineage differentiation. Through live imaging and biochemical studies, we find that loss of Krill causes the accumulation of misfolded proteins and excessive PERK activation-dependent autophagy in HSPCs. Blocking autophagy but not inhibiting apoptosis by Bcl2 overexpression can fully rescue hematopoietic defects, but not the lethality of $k$ rill $^{\text {cas } 002}$ embryos. Treatment with autophagy inhibitors (3-MA and Baf A1) or PERK inhibitor (GSK2656157), or knockdown of beclin1 or perk can markedly restore HSPC proliferation and definitive hematopoietic cell differentiation. These results may provide leads for effective therapeutics that benefit patients with anemia or bone marrow failure caused by ribosome disorders.

Keywords: Hematopoietic stem cells; zebrafish; krill; ribosome biogenesis; autophagy; PERK; misfolded/unfolded protein Cell Research (2015) 25:946-962. doi:10.1038/cr.2015.81; published online 3 July 2015

*These three authors contributed equally to this work.

Correspondence: Weijun Pan ${ }^{\mathrm{a}}$, Saijuan Chen ${ }^{\mathrm{b}}$, Yi Zhou

${ }^{a}$ E-mail: weijunpan@sibs.ac.cn

${ }^{b}$ E-mail: sjchen@stn.sh.cn

'E-mail: yzhou@enders.tch.harvard.edu

Received 12 January 2015; revised 3 May 2015; accepted 28 May 2015; published online 3 July 2015

\section{Introduction}

Vertebrate hematopoiesis is an evolutionarily conserved and highly regulated process involving the production of differentiated blood cell lineages from hematopoietic stem cells (HSCs) [1-3]. The zebrafish (Danio rerio) is a powerful genetic and developmental model to study the development of vertebrate circulatory system, 
especially hematopoiesis [4, 5]. Definitive hematopoiesis in zebrafish is highly conserved with mammals [6]. It occurs at the ventral wall of dorsal aorta in a structure called aorta-gonad-mesonephros (AGM) around 28 hour post-fertilization (hpf) [7]. By 2 days post-fertilization (dpf), the AGM-derived HSCs migrate to caudal hematopoietic tissue (CHT), an embryonic structure analogous to the mammalian fetal liver [8-10], for rapid pool expansion and lineage differentiation.

Normal ribosome biogenesis is critical for cell survival, proliferation and function. In eukaryotic cells the small subunit (SSU) processome, a ribonucleoprotein complex, plays essential roles in $18 \mathrm{~S}$ rRNA maturation, small ribosomal subunit assembly and subsequent ribosome biogenesis [11]. The SSU processome is composed of pre-rRNA, small nucleolar RNAs and more than 70 different associated proteins. Dysfunction of ribosome biogenesis is associated with human diseases, including Diamond-Blackfan anemia (DBA), 5q minus (del (5q-)) syndrome, dyskeratosis congenita and Bowen-Conradi syndrome [12-14]. In addition to carrying genetic mutations affecting ribosome biogenesis, these human diseases share same clinical features, and are thus termed as "ribosomopathies" [13].

Previous works have shown that upregulation of p53 by ribosome dysfunction contributes to bone marrow failure syndromes [15] that have been genetically mapped to loss-of-function mutations in rps14 [16], rps19 [17, 18], rpl11 [19] and rps29 [15]. The apoptotic phenotype of hematopoietic cells in these diseases can be partially restored by p53 downregulation $[16,17]$. However, some studies have reported that p53 pathway is not involved in some ribosome biogenesis disorders [20-22]. Furthermore, not all bone marrow samples from patients with del $\left(5 \mathrm{q}^{-}\right)$syndrome or DBA show p53 activation, suggesting that other mechanisms may contribute to ribosomopathies [23]. For example, loss of $p w h p 2 h$ causes gut degeneration and hyperactivated autophagy in a p53and mTOR-independent manner [21]. Knockdown of Rp122 in zebrafish embryos blocks T-lineage progenitor development, while knockdown of the Rpl22 paralog Rpl221 impairs the emergence of HSC in AGM by abrogating Smad1 expression and Runx1 induction [24].

Autophagy and apoptosis are two major stress-response pathways. Dysregulation of autophagy has been linked to many human diseases such as neurodegeneration $[25,26]$, autoimmunity and cancer [27, 28]. Multiple upstream signaling mechanisms, including mTOR pathway, unfolded protein response (UPR), ER stress and nutrition stress regulate autophagy, with Beclin1-VPS34 complex playing an important role in autophagy initiation $[29,30]$. Autophagy is a critical mechanism that pro- tects HSCs from stress damages [31]. In mice, a conditional deletion of atg7 in HSCs renders the loss of HSC's self-renewal property and severe myeloproliferation due to a failure of HSPCs to respond normally to stress from reactive oxygen species (ROS) [32]. Appropriate autophagy level is also important for lymphocyte survival [33, 34] and erythroid cell maturation [35-37]. Patients with certain ribosomopathies have elevated levels of autophagy in peripheral blood cells resulted from S6K-induced inhibition on insulin pathway activation [38]. However, the potential cures for these disorders have not been found.

In the present study, we report that krill gene is essential for definitive hematopoiesis. Loss of Kri11, a critical component of SSU complex, causes ribosomal biogenesis defects, accumulation of misfolded proteins and activation of PERK-eif2a signaling. These deficiencies subsequently hyperactivate autophagy and ultimately lead to the inhibition of HSPC proliferation. Treatment with autophagy or PERK inhibitors, or knockdown of beclin1 or perk by morpholino (MO), can successfully rescue HSPC proliferation and lineage differentiation in krill mutant.

\section{Results}

cas002 mutant displays a hematopoietic failure phenotype

In a large-scale ENU mutagenesis screen for definitive hematopoietic mutations, we obtained cas002, a novel mutant with severe hematopoietic defects and recessive lethality. cas002 embryos are morphologically indistinguishable from wild-type siblings before $3 \mathrm{dpf}$, with normal blood flow and heart beats (Figure 1A-1B). However, whole-mount in situ hybridization (WISH) of $c m y b$ reveals a markedly reduced HSPC population in caudal hematopoietic tissue (CHT) of mutant embryos at $3 \mathrm{dpf}$ (Figure 1C-1D), and in CHT, thymus and kidney at $5 \mathrm{dpf}$ (Figure 1E-1F). cas002 mutant embryos eventually die at 6-10 dpf with abnormal head shape, cardiac edema and smaller eyes.

To examine hematopoiesis phenotype in detail, we performed WISH analysis of different cell lineage markers: gatal (erythrocyte progenitors), ael-globin (embryonic erythrocytes), l-plastin (pan-myeloid cells), lyz and mpo (neutrophils). The expression of these markers is the same as wild-type siblings at $3 \mathrm{dpf}$ (Supplementary information, Figure S1), but becomes significantly reduced in cas002 mutant embryos at $5 \mathrm{dpf}$ (Supplementary information, Figure S2A-S2J). The expression of ragl (T lymphocyte), is substantially reduced at $4 \mathrm{dpf}$ in cas002 (Supplementary information, Figure S2K-S2L), 

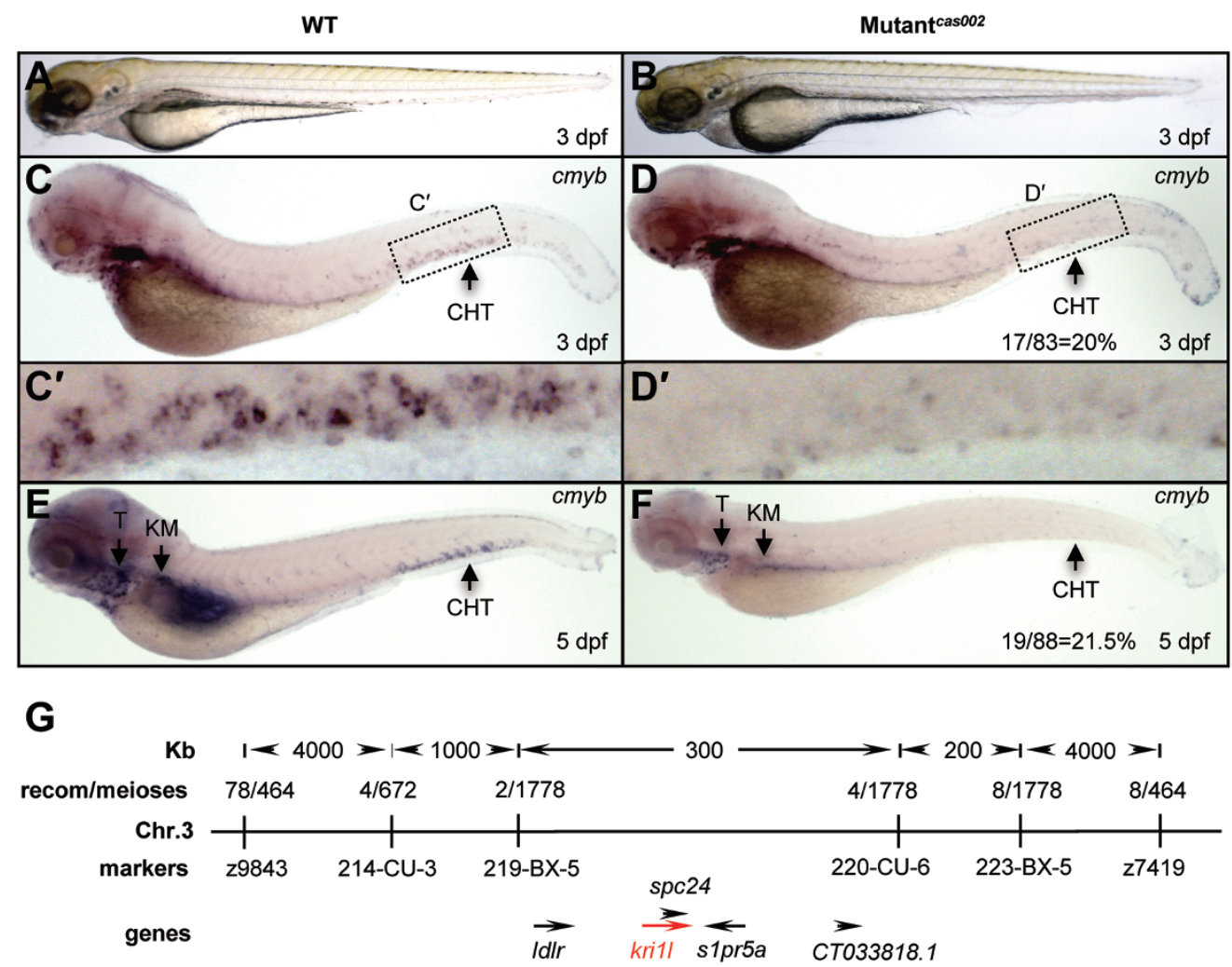

H

WT GAGAAGTATGAGAAATATCGCCAGAAAGAGgAACTGCAGATACTGAAG

MU GAGAA

-TGAAG

I

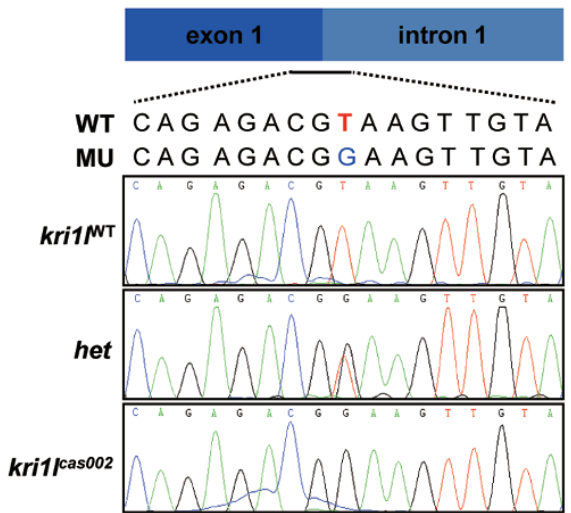

J

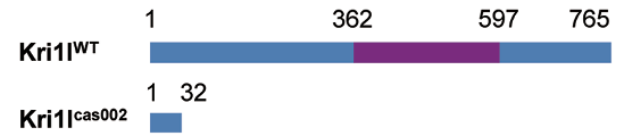

K

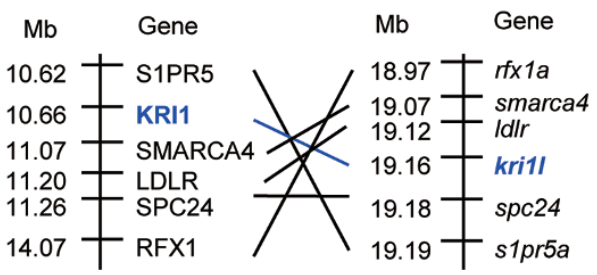

Human chr.19

Figure 1 Hematopoietic defects and positional cloning of cas002 mutant. (A-B) Light microscope images of zebrafish wildtype (WT) and cas002 embryos at $3 \mathrm{dpf}$. (C-F) WISH analysis of cmyb expression in WT and cas002 embryos at indicated development stages. Black arrows indicate thymus, kidney marrow and CHT. (C'-D') enlarged CHT regions in $\mathbf{C}$ and $\mathbf{D}$. (G) Genetic mapping of the cas002 region on chromosome 3. Bulk segregation analysis locates cas002 mutation to Chr. 3 . Fine mapping using SSLPs narrows down to a region between markers 219-BX-5 and 220-CU-6, containing kri1/ and four other genes as indicated. $(\mathrm{H})$ The sequencing results of kri1/ cDNA from mutant embryos show a 38 bp deletion (MU) compared with kri1/ cDNA from WT embryos. (I, J) The sequencing result of kri1l genomic DNA shows a T-G transversion at the kri1l exon 1-intron 1 consensus splicing donor site $(\mathbf{I})$, which causes a frame shift $(\mathbf{H})$ and a premature stop codon leading to the production of a truncated Kri1l protein (J). (K) Synteny between zebrafish kri1/ I and human KRI1 loci. (Left) Six genes, including $K R / 1$, are located within a genomic region on human chromosome 19. (Right) Six zebrafish homologs are listed according to their map positions on chromosome 3 (Ensembl website). CHT, caudal hematopoietic tissue; KM, kidney marrow; $\mathrm{Mb}$, mega base; $\mathrm{T}$, thymus; het, heterozygote. 
indicating that lymphoid development is also impaired. In contrast, primitive hematopoiesis and vascular morphogenesis are intact in cas002 embryos (Supplementary information, Figure S3). To identify the onset of hematopoiesis failure in cas002 mutant embryos, we traced expression of cmyb and runx1, another HSC marker, at earlier developmental time points (Supplementary information, Figure S4). Both cmyb and runxl are expressed normally in the AGM from $36 \mathrm{hpf}$ to $40 \mathrm{hpf}$ (Supplementary information, Figures S4A-S4D and S4C'-S4D') and at $36 \mathrm{hpf}$ (Supplementary information, Figure S5AS5B), respectively, suggesting a normal hematopoiesis in the AGM at these stages. A marginal decrease of $c m y b$ expression is detectable in the CHT region at $40 \mathrm{hpf}$ (Supplementary information, Figure S4C"-S4D"). By $4 \mathrm{dpf}$, the expression of cmyb is almost undetectable in the CHT, kidney and thymus of cas002 mutant embryos (Supplementary information, Figure S4K-S4L). Another HSPC marker $s c l$ is also markedly reduced from $3 \mathrm{dpf}$ (Supplementary information, Figure S5C-S5H). In addition, cas002 mutant embryos have significantly fewer EGFP $^{+}$cells (marked by eGFP under $c m y b$ promoter) than the wild type (Supplementary information, Figure S6A-S6G). Taken together, these results suggest that definitive HSPCs in the CHT are severely disrupted in cas002 mutant embryos.

\section{cas002 mutant carries a defective krill gene}

To understand the mechanism of hematopoietic failure in cas002 mutant, we carried out positional cloning [39]. The mutation was first mapped on chromosome 3 by bulk segregation analysis (BSA). Simple sequence length polymorphism (SSLP)-based fine mapping established that the mutation lies within a $300 \mathrm{~kb}$ region between two markers: 219-BX-5 and 220-CU-6 (Figure 1G). We sequenced all five candidate genes in this region, and found a $38 \mathrm{bp}$ deletion in krill cDNA in cas002 mutant (Figure 1H). Genomic DNA sequencing of krill gene revealed that a consensus splicing donor site at the boundary between exon 1 and intron 1 is disrupted by a T-to-G transversion in cas002 mutant (Figure 1I). This mutation yields an abnormally spliced transcript with a frame shift and a premature stop codon in exon2. The altered transcript is predicted to encode a highly truncated Krill peptide 32 amino acid in length (Figure 1J). This point mutation is not found among five commonly used laboratory zebrafish strains (Tu, AB, WIK, Longfin and Shanghai; Supplementary information, Figure $\mathrm{S} 6 \mathrm{H}$ ), excluding the possibility of single-nucleotide polymorphism [40]. The zebrafish krill gene is $62 \%$ identical with the human KRII. The krill locus on zebrafish chromosome 3 is syntenic to a region of human chromosome 19 that con- tains the KRI1 gene, based on the conserved locations of neighboring orthologous gene pairs (S1PR5, SMARCA4 and $L D L R$; Figure $1 \mathrm{~K}$ ). These findings suggest that zebrafish krill gene is an ortholog of human KRI1.

\section{Mutated krill causes cas002 phenotypes}

To confirm that the mutation in krill gene is responsible for cas002 phenotypes, we microinjected krill ATG morpholino (MO) and splicing MO to suppress translation and maturation of krill mRNA, respectively, into wild-type zebrafish embryos. A construct containing a 60-bp fragment including krill ATG MO-binding site fused to the N-terminus of EGFP (Supplementary information, Figure S7E) was co-injected with krill ATG MO; the expression of EGFP was successfully blocked (Supplementary information, Figure S7A-S7D). The krill splicing MO caused splicing defect in the endogenous krill transcripts (Supplementary information, Figure S7F). cmyb expression and $\mathrm{EGFP}^{+}$cell numbers in both morphants in Tg (cmyb:egfp) background were significantly reduced, indicating that krill knockdown reproduces hematopoietic defects found in cas002 mutant (Figure 2A-2C and Supplementary information, Figure S7G-S7H).

To provide further evidence that krill is defective in cas002 mutant, we performed a rescue experiment by microinjection of synthetic wild-type krill mRNA into cas002 mutant embryos. As monitored by WISH analysis of $c m y b$ expression, we found that the definitive hematopoiesis was fully rescued by wild-type Krill overexpression (Figure 2D-2F). In summary, results from positional cloning, MO phenocopy and mRNA rescue experiments strongly suggest that the T-to-G mutation in zebrafish krill gene is responsible for the defective hematopoiesis in cas002 mutant embryos. We thus rename the mutant as $k r i 1 l^{\text {cas } 002}$.

To understand the role of krill in embryogenesis, especially in definitive hematopoiesis, we examined the temporal and spatial expression of krill by WISH analysis. The krill transcript is expressed as maternal mRNA. During early development, krill is expressed ubiquitously throughout the embryo and enriched in somites at $18 \mathrm{hpf}$ and eyes at $22 \mathrm{hpf}$ (Supplementary information, Figure S8).

In yeast, KRI1 is a component of the SSU complex, and plays an essential role in $40 \mathrm{~S}$ ribosome subunit formation and ribosomal polysome assembly. Mechanistically, loss of KRI1 results in instability of $18 \mathrm{~S}$ rRNA precursor and dramatic reduction of mature 18S rRNA [41]. We asked whether krill ${ }^{\text {cas } 002}$ mutant embryos have similar defects in ribosome biogenesis. E-bioanalyser analysis of total RNA revealed a dramatic reduction in 

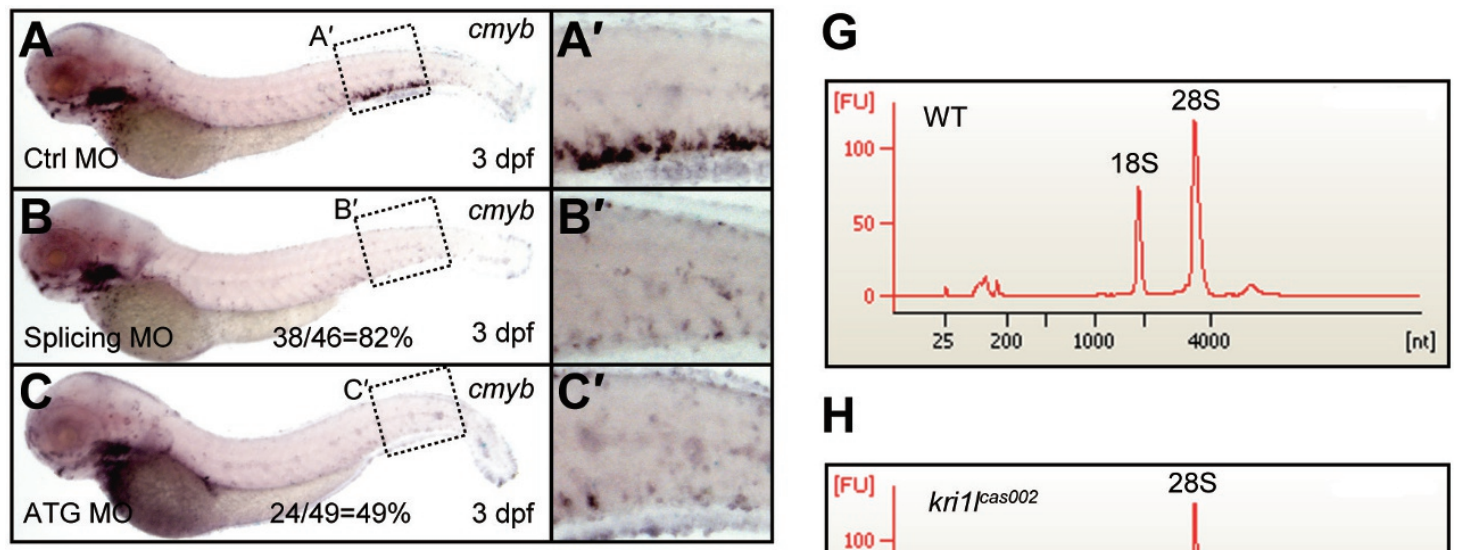

\section{H}
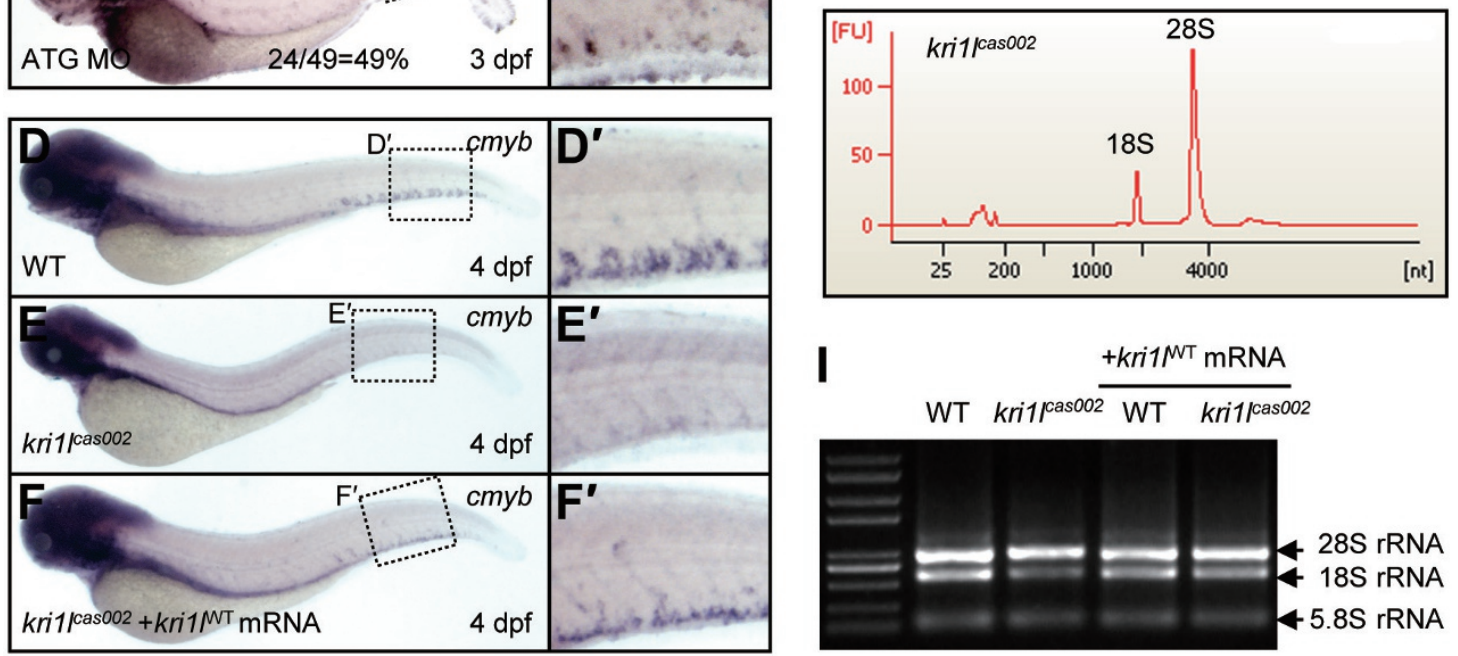

Figure 2 Mutation in kri1/ gene is responsible for cas002 phenotypes. (A-C) Morpholino knockdown of kri1/ phenocopies cas002 mutant. kri1/ ATG MO and splicing MO were injected into wild-type embryos at one-cell stage. At $3 \mathrm{dpf}$, the injected embryos were fixed and analyzed for cmyb expression by WISH. The percentage of morphants with the reduced cmyb expression phenotype is listed at the bottom of B and C. (D-F) Transient expression of wild-type kri1/ mRNA rescues cas002 mutant. cas002 mutant embryos at one-cell stage were injected with synthesized wild type kri1/ mRNA. The injected embryos were fixed for analysis of cmyb gene expression using WISH at $4 \mathrm{dpf}$. After WISH and photographing, all embryos were genotyped by sequencing of genomic DNA; the percentage of the rescue was then evaluated. The percentage of fully rescued mutant embryos is about 54\% (50/92), while the rest are partially rescued. (A'-F') Details of cmyb expression in CHT regions in A-F. (G-H) E-Bioanalyser analysis of total RNA isolated from WT and kri1/ casoo2 embryo pools (each pool of 12 embryos) at $3 \mathrm{dpf}$. A significant reduction in the $18 \mathrm{~S}$ peak but unchanged $28 \mathrm{~S}$ peak in kri1 ${ }^{\text {cas } 002}$ results in an elevated $28 \mathrm{~S} / 18 \mathrm{~S}$ rRNA ratio. (I) The $18 \mathrm{~S}$ rRNA reduction can be restored in mutant embryos by injection of $k r i 1{ }^{\mathrm{NT}}$ mRNA.

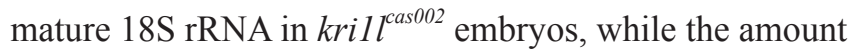
of 28S rRNA was normal (Figure 2G-2H), which is consistent with previous observations in KRI1-deficient yeast cells. Reduced 18S rRNA could be restored by microinjection of wild-type krill mRNA into krill $l^{\text {cas } 002}$ mutants (Figure 2I). We further examined the total protein level by bicinchoninic acid (BCA) protein quantitation and confirmed impaired protein synthesis in krill $l^{\text {cas } 002}$ mutant embryos (Supplementary information, Figure S9A-S9C) $(P=0.0003)$. Sequencing of $18 \mathrm{~S}$ rRNA in wild-type and $k_{\text {ril }}$ cas 002 mutant embryos showed no difference; however, $80 \mathrm{~S}$ ribosome and polysome formation were impaired in $\mathrm{krill}^{\mathrm{cas} 002}$ mutant embryos with excessive 60S ribosome subunits (Supplementary information, Figure S9D).
Bcl2 rescues hematopoiesis in krill ${ }^{\text {cas } 002}$ mutant

To elucidate the mechanism of defective HSPCs in krill mutant, we examined HSPC proliferation by calculating the proportion of phospho-histone $3(\mathrm{pH} 3)$ immunostaining-positive cells in total HSPC (cmyb WISH positive) during hematopoietic development (Figure 3A, 3F, 3G-3R and Supplementary information, Figure S10). At $40 \mathrm{hpf}$, the percentage of proliferative HSPCs $\left(\mathrm{pH}^{+}{ }^{+}\right.$$m y b^{+} / \mathrm{cmyb}^{+}$) in $k r i 1 l^{\text {caso02 }}$ mutant embryos was nearly the same as that in wild-type siblings in the AGM (Figure 3A and Supplementary information, Figure S10A-S10H; $P=$ 0.0873 ), but was significantly reduced in the CHT (Figure $3 \mathrm{~A}$ and Supplementary information, Figure S10I-S10P; $P<0.0001$ ), and was further decreased in the CHT at 2 
A
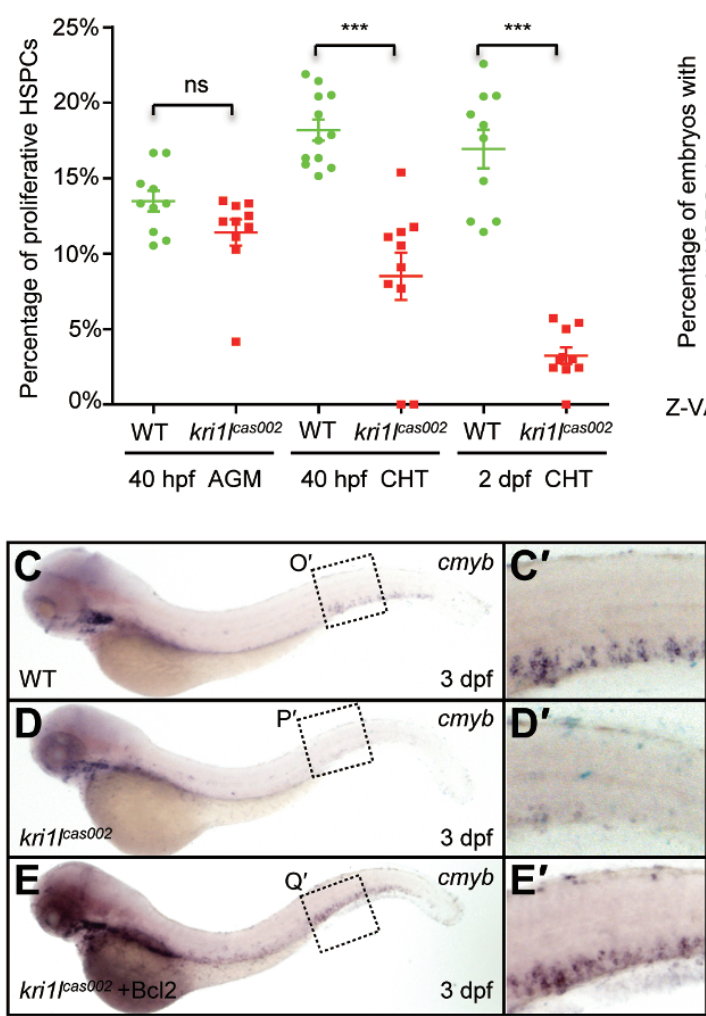

B

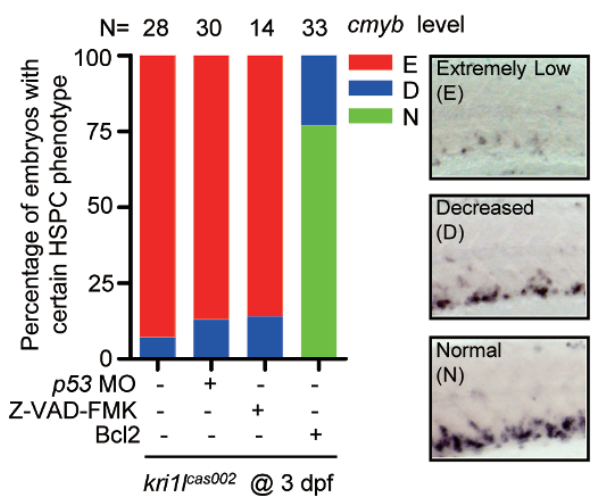

$\mathbf{F}$

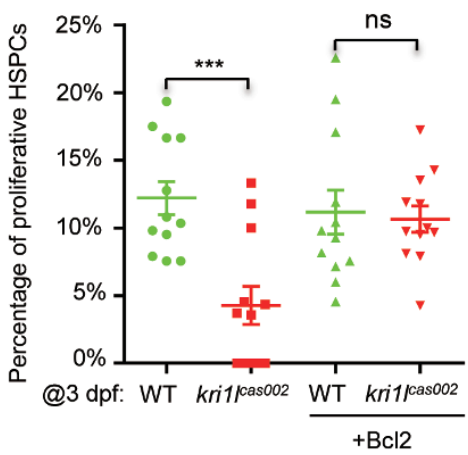

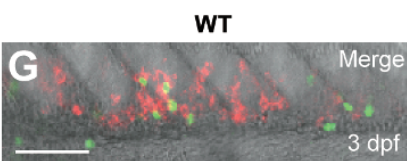
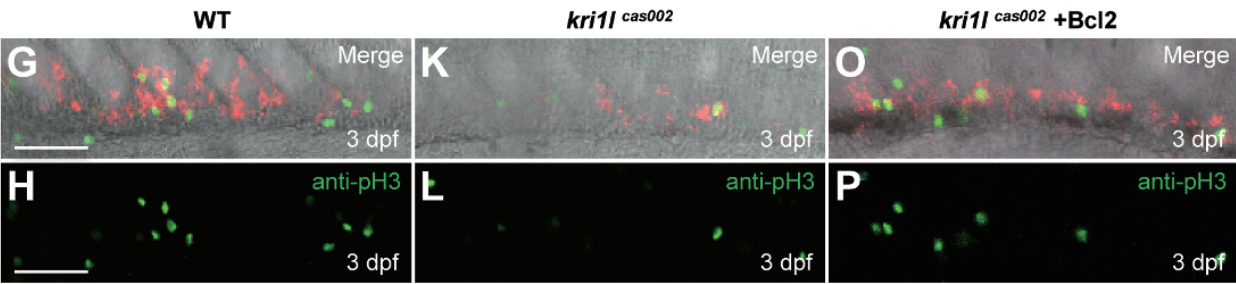

anti-pH3
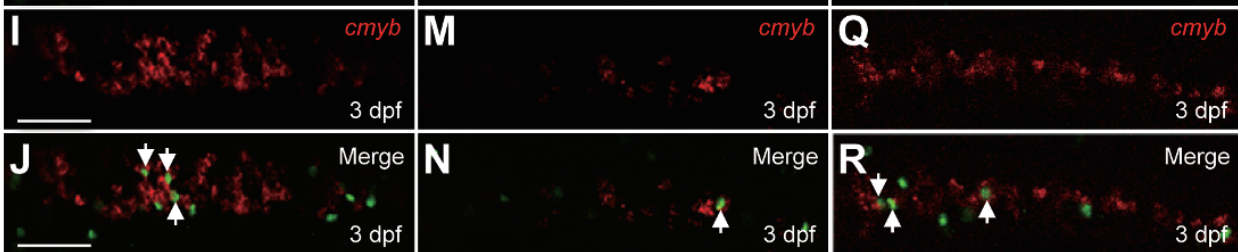

Figure 3 Transient expression of Bcl2 rescues the defective hematopoiesis in kri1/ ${ }^{\text {cas } 002}$ mutant embryos. (A) Statistic analysis of the proportion of proliferative HSPCs (cmyb and PH3 double positive) in total HSPCs in kri1/ ${ }^{\text {cas } 002}$ mutant embryos and wildtype siblings during hematopoietic development (40 hpf-2 dpf). N $\geq 10$ for each group. $40 \mathrm{hpf}$ AGM, $p=0.0873 ; 40 \mathrm{hpf} \mathrm{CHT}$, $p<0.0001 ; 2 \mathrm{dpf} \mathrm{CHT}, p<0.0001$. Error bars represent SEM. ${ }^{* *} P \leq 0.01$; ${ }^{* \star} P \leq 0.001$ (Student's $t$-test). (B) Proportion of $k_{r i 1 l^{c a s} 002}$ mutant embryos with classified hematopoiesis phenotype (indicated by cmyb staining) with or without microinjection of p53 MO, treatment with caspase inhibitor (Z-VAD-FMK) or Bcl2 expression, respectively. (C-E) The CHT hematopoiesis is recovered by $\mathrm{Bcl} 2$ overexpression in $\mathrm{kri1}^{\mathrm{cas} 002}$ mutant embryos. Embryos from two heterozygous parents were injected with bc/2 mRNA at one-cell stage and harvested for WISH analysis. After WISH and photographing, all embryos were genotyped by genomic DNA sequencing; percentage of rescue was then calculated. 26 out of 33 mutant embryos injected at one-cell stage with $b c / 2$ mRNA are fully rescued, while the rest of embryos are partially rescued. (C'-E') The enlarged CHT regions. (F) Percentage of the proliferative HSPCs in the $\mathrm{CHT}$ of kri1/ ${ }^{\text {cas } 002}$ mutant embryos and wild-type siblings with or without Bcl2 overexpression at $3 \mathrm{dpf}$. $N=12$ for each group. Without $\mathrm{Bcl} 2$ overexpression, $p<0.0001$; With $\mathrm{Bcl} 2$ overexpression, $p=0.3136$. Error bars represent SEM. ${ }^{* *} P \leq 0.01$; ${ }^{* *} P \leq 0.001$ (Student's $t$-test). (G-R) Representative confocal images of double staining of cmyb RNA (red) and pH3 protein (green) in the $\mathrm{CHT}$ at $3 \mathrm{dpf}$. $\mathbf{G}, \mathbf{K}$ and $\mathbf{O}$ are bright-field images overlaid with fluorescent staining. Scale bars, $50 \mu \mathrm{m}$. 
dpf (Figure 3A and Supplementary information, Figure S10Q-S10X; $P<0.0001)$ and $3 \mathrm{dpf}$ (Figure 3F-3R) $(P=$ 0.0003).

Previous reports have demonstrated that p53-dependent apoptosis pathway is activated and responsible for hematopoietic defects in both ribosomopathy-related mice and zebrafish models [15-18]. We carried out terminal transferase dUTP nick end labeling (TUNEL) assays in wild-type siblings and $\mathrm{krill}^{\mathrm{cas} 002}$ mutant embryos from $40 \mathrm{hpf}$ to $3 \mathrm{dpf}$; however, there was no significant difference in apoptotic signals in the CHT region between two genotypes (Supplementary information, Figure S11).

Furthermore, we microinjected p53 MO [42] into krill $^{\text {cas } 002}$ mutant embryos (Figure 3B and Supplementary information, Figure S12A-S12D), and kri1l MO into p5 $3^{M 214 K}$ mutant embryos (Supplementary information, Figure S13A-S13D). The defective hematopoiesis remained in these morphants, suggesting that p53-dependent apoptosis is not responsible for the hematopoietic failure in $\mathrm{krill}^{\mathrm{cas} 002}$ mutant. We further tested whether the activation of caspase pathway contributes to HSPC defects and found treatment of pan-caspase inhibitor, Z-VAD-FMK peptide, could not rescue krill $l^{\text {cas } 002}$ phenotype (Figure 3B and Supplementary information, Figure S12E-S12F). Quantitative PCR and immunoblotting analysis also showed that $\Delta 113-\mathrm{p} 53$, an inhibitor of fulllength p53 [43], but not full-length p53, was dramatically upregulated in krill ${ }^{\text {cas } 002}$ mutant embryos (Supplementary information, Figure S14).

In parallel, we overexpressed an EGFP-Bcl2 fusion protein in krill $l^{\text {cas } 002}$ mutant embryos. To our surprise, microinjection of egfp-bcl2 mRNA fully restored $c m y b$ expression in the CHT of krill cas002 mutant embryos at 3 dpf (Figure 3B-3E). We performed WISH analysis of $\alpha e 1$-globin and lyz expression at $4 \mathrm{dpf}$, and found $\mathrm{Bcl} 2$ overexpression also successfully restored the erythrocytes (74\%; Supplementary information, Figure S15AS15D) and neutrophils (61\%; Supplementary information, Figure S15E-S15H). Very importantly, ectopic expression of $\mathrm{Bcl} 2$ almost fully restored the percentage of proliferating HSPCs (Figure 3F-3R; $P=0.3136$ ) in krill las $^{\text {cas }}$ mutant embryos.

\section{Autophagy is hyperactivated in krill cas002 mutant}

Recent reports have shown that $\mathrm{Bcl} 2$ functions not only as an anti-apoptosis protein, but also as an anti-autophagy protein by interacting with Beclin1 and disrupting VPS34-Beclin1 complex [44, 45]. To check whether the regulatory role of $\mathrm{Bcl} 2$ in autophagy is responsible for the rescue of HSPC in kril cas002 mutant, we carried out immunoblotting on the whole embryo lysates using an antibody against an autophagy marker, microtubule-associated protein light chain 3 (Lc3) $[46,47]$. We found the level of Lc3-II in krill $l^{\text {cas002 }}$ mutant was significantly higher than that in wild-type embryos (Figure 4A), and it could not be rescued in $p 53^{M 214 K}$ mutant background [48] (Supplementary information, Figure S13E). In addition, we found that the level of p62 protein [49], a well-characterized autophagy substrate, was decreased in $\mathrm{krill}^{\text {cas } 002}$ mutant embryos, while the level of autophagy initiation factor Beclin1 was unchanged in the mutant embryos (Figure 4A and Supplementary information, Figure S14A).

In order to directly observe the autophagy level in live krill $l^{\text {cas } 002}$ mutant $[46,50]$, we injected a $m$ Cherry-lc3 fusion RNA (in vitro transcribed from a previously described construct [51]) into wild-type or krill $l^{\text {cas } 002}$ embryos transgenic for cmyb:egfp at one-cell stage. At $3 \mathrm{dpf}$, abundant Lc3-II puncta (indicating autophagosomes) were present in krill $l^{\text {cas } 002} \mathrm{EGFP}^{+}$cells in the CHT (Figure 4B-4H; $P=0.004)$, and this phenomenon became more obvious after chloroquine treatment, which is known to block the degradation of autophagosome $[52,53]$ (Figure 4B, 4I-4N; $P=0.0008$ ). The increased Lc3-II puncta in HSPC could be found as early as $40 \mathrm{hpf}$ in the CHT of $k_{\text {rill }}^{\text {cas } 002}$ mutant (Supplementary information, Figure S16). Analysis of electron micrographs further confirmed a significant increase of autophagosome-like structures above the wild-type level in the CHT region of krill cas002 mutant (Supplementary information, Figure S17). These results suggest that the autophagy level is significantly elevated in $\mathrm{kri}_{1} l^{\mathrm{cas} 002}$ mutant, and this change may correlate with the reduction of HSPCs.

To test whether $\mathrm{Bcl} 2$ overexpression rescues the hematopoietic failure in krill cas002 mutant embryo through inhibiting autophagy, we evaluated the Lc3-II level in $\mathrm{krill}^{\mathrm{cas} 002}$ mutant with or without overexpression of Bcl2. Consistent with our hypothesis, $\mathrm{Bcl} 2$ overexpression significantly reduced the Lc3-II level in krill $l^{\text {cas } 002} \mathrm{mu}-$ tant (Figure 4O). mCherry-Lc3-labeled autophagosomes in $\mathrm{EGFP}^{+}$HSPCs were also markedly decreased in Bcl2-overexpressed krill $l^{\text {cas } 002}$ mutant (Figure 4P-4S; $P=$ 0.0021 ), suggesting Bcl2 rescues kril cas 002 hematopoietic phenotype through its anti-autophagy function.

Inhibition of autophagy restores hematopoiesis in krill cas002 $^{2}$ mutant

Autophagy inhibitors have been successfully developed to target different stages of autophagic flux. We hypothesized that the treatment with these inhibitors, or knockdown of the autophagy initiation factor Beclin1, might mimic the effect of $\mathrm{Bcl} 2$ overexpression in restoring hematopoiesis in krill ${ }^{\text {cas } 002}$ mutant. Indeed, $\mathrm{krill}^{\text {cas } 002}$ mutant embryos treated with 3-MA [54] at $10 \mathrm{mM}$ or Bafilomycin A1 (Baf A1) [55] at $25 \mathrm{nM}$ for $36 \mathrm{~h}$, or injected with beclin1 MO at one-cell stage, showed normal 
A
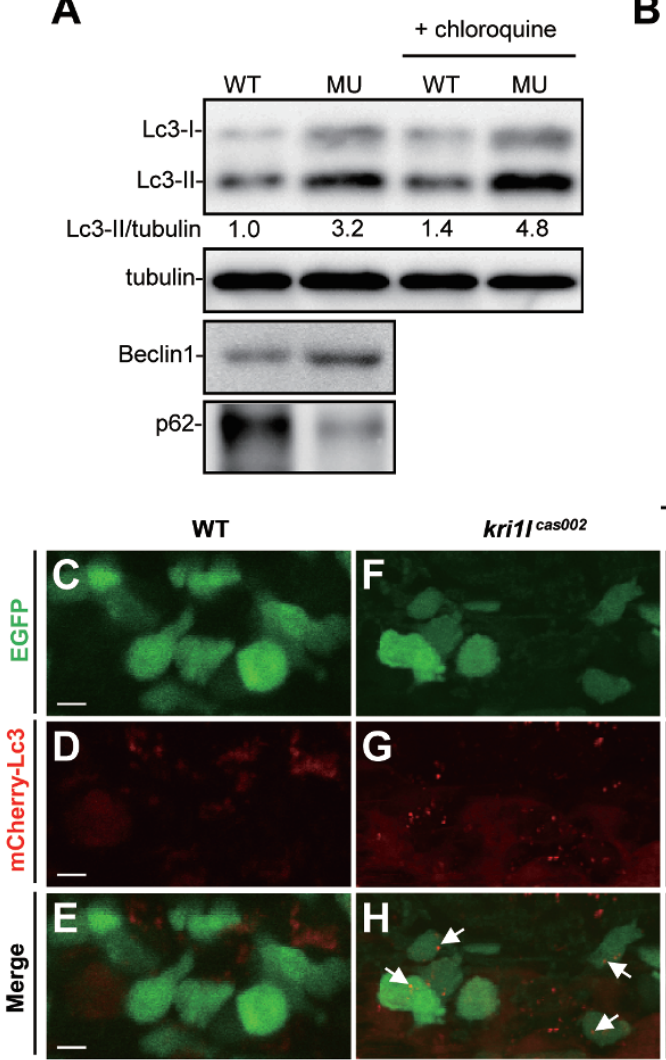

0

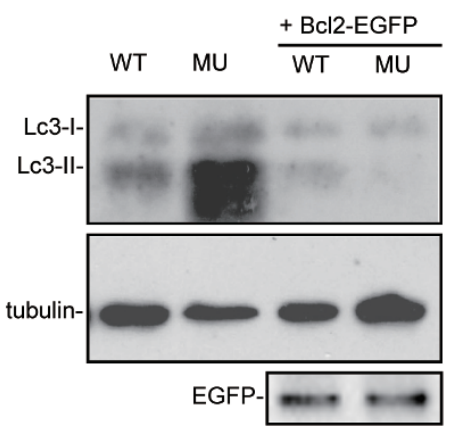

B

$\mathbf{P}$
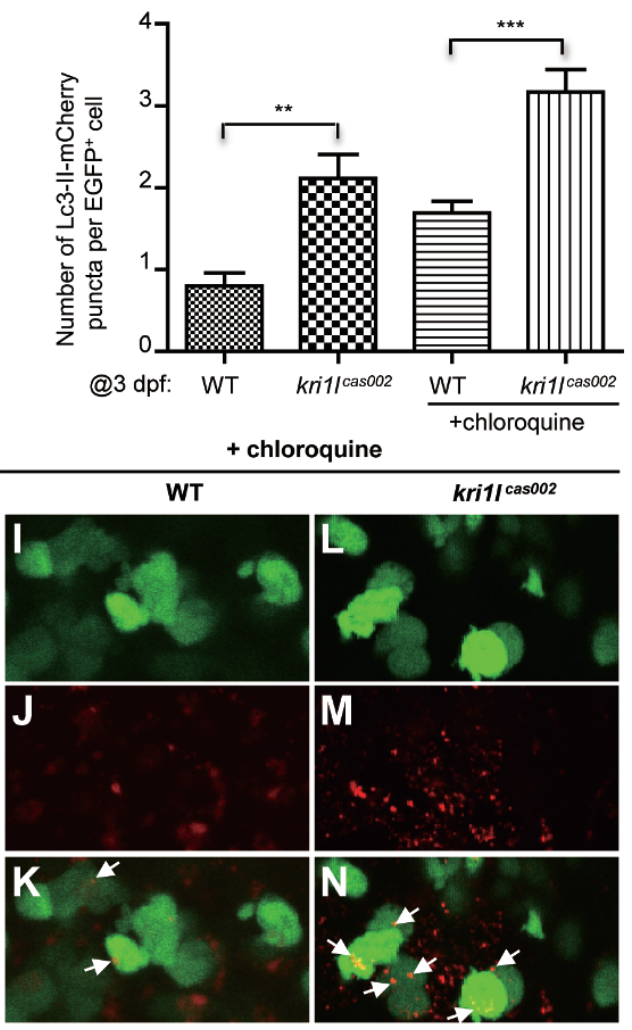

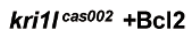
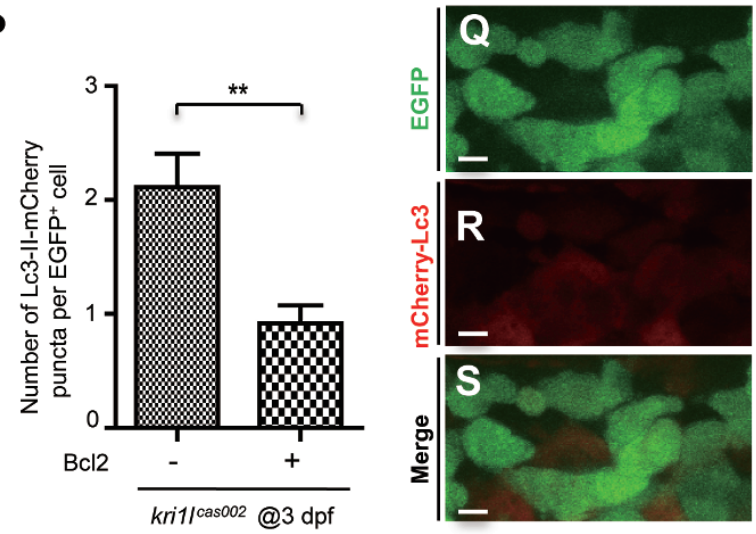

Figure 4 Ectopic expression of Bcl2 inhibits excessive autophagic flux in kri1/ ${ }^{\text {cas002 }}$ HSPCs. (A) Representative immunoblotting images of Lc3-I, Lc3-II, Beclin1 and p62 in whole embryo lysates of wild-type (WT) and kri1/ cas002 embryos at 3 dpf, treated with or without chloroquine $(5 \mu \mathrm{M})$ for $6 \mathrm{~h}$ before harvest. Tubulin serves as the loading control. (B) Statistics of the average number of autophagosome (indicated by mCherry-Lc3 puncta) in kri1 ${ }^{\text {cas } 002}$ mutant embryos and WT siblings with or without chloroquine treatment at $3 \mathrm{dpf}$. mCherry-Lc3 puncta are counted in over 20 EGFP $^{+}$cells (in cmyb:egfp background) per embryo, total 6-8 embryos under each condition. Without chloroquine treatment, $P=0.0040$; with chloroquine treatment, $P=0.0008$. Error bars represent SEM. ${ }^{* *} P \leq 0.01 ;{ }^{* *} P \leq 0.001$ (Student's $t$-test). (C-N) Representative confocal images of mCherry-Lc3 puncta (autophagosomes) in HSPCs of live zebrafish embryos. Wild type siblings or kri1/ ${ }^{\text {cas } 002}$ mutant embryos in the Tg (cmyb: egfp) transgenic background, were injected with mcherry-lc3 mRNA at one-cell stage and imaged at $3 \mathrm{dpf}$ with or without pre-treatment with chloroquine $(5 \mu \mathrm{M})$ for $6 \mathrm{~h}$. Scale bars: $5 \mu \mathrm{m}$. (O) Immunoblotting analysis of whole embryo lysates from WT and kri1/ $l^{\text {cas } 002}$ mutant embryos with or without $b c / 2$ mRNA injection. (P) Statistics of the average number of autophagosome for kri1 ${ }^{\text {ras } 002}$ mutant embryos and wild type siblings with Bcl2 overexpression at $3 \mathrm{dpf}$. mCherry-Lc3 puncta are counted in over $20 \mathrm{EGFP}^{+}$(cmyb: egfp) cells per embryo, total 8-11 individual embryos. $P=0.0021$. Error bars represent SEM. ${ }^{* *} P \leq 0.01$; ${ }^{* * *} P \leq 0.001$ (Student's $t$-test). (Q-S) Representative confocal images of mCherry-Lc3 puncta (autophagosomes) in HSPCs of live zebrafish embryos with Bcl2 overexpression. Scale bars, $5 \mu \mathrm{m}$. 
cmyb expression in the CHT (Figure 5A-5E). 3-MA significantly reduced autophagy level in kri $1 l^{\text {cas } 002}$ HSPCs, which was visualized by live imaging and quantitation of Lc3-II puncta (Figure 5F-5L; $P=0.0037$ ). Furthermore, both Baf A1 (Figure 5M-5U) and 3-MA (data no shown) treatment could restore $\mathrm{krill}^{\text {cas } 002} \mathrm{HSPC}$ proliferation. At 3 dpf, the proportion of $\mathrm{pH}^{+} \mathrm{Cmyb}^{+}$cells in $\mathrm{krill}^{\mathrm{cas} 002}$ mutant embryos treated with Baf A1 was almost the same as that in wild type (Figure 5U; $P=0.2344$ ). Therefore, inhibition of autophagic flux at different stages is able to rescue HSPC proliferation defects in $\mathrm{krill}^{\text {cas } 002}$ mutant.

Accumulation of misfolded proteins trigger PERK-dependent autophagy

To understand how Krill deficiency-caused ribosome defects trigger excessive autophagy via a p53-independent manner, we hypothesized that impaired ribosome biogenesis might cause dysfunction in protein synthesis to trigger misfolded protein accumulation, which is known to activate autophagy [56, 57].

Protein remodeling factor Hsp110 is known to cooperate with Hsp70 and Hsp40 to dissolve and reactivate aggregated proteins. It is used as a molecular probe to detect puncta foci where misfolded proteins accumulate [58]. We injected an in vitro transcribed mRNA coding for Hspa4a (zebrafish homolog of human Hsp110) [59] fused to mCherry into one-cell stage wild-type or krill $l^{\text {cas002 }}$ embryos stably transgenic for cmyb:egfp. Abundant Hspa4a-mCherry puncta were observed in krill $l^{\text {cas } 002}$ $\mathrm{EGFP}^{+}$cells in the CHT region (Figure 6A-6G; $P=$ 0.0002 ), indicating the accumulation of misfolded proteins in Krill deficient HSPCs.

Ubiquitination is an important protein post-translational modification, which governs protein quality control. Misfolded or unfolded proteins contain more lysine residues on their surface, and are prone to be recognized and subjected to ubiquitination and degradation [60]. Microinjection of Flag-tagged ubiquitin-expressing construct was performed with or without krill ATG MO. We found a dramatic accumulation of ubiquitin-modified proteins in krill ATG morphant (Figure 6H).

Accumulation of misfolded/unfolded proteins is known to trigger activation of PERK, which subsequently phosphorylates eif2a to inhibit protein synthesis [61]. Immunoblotting analysis showed that the phosphorylation level of eif2a protein was indeed upregulated in krill $l^{\text {cas } 02}$ mutant (Figure 6H). To assess whether PERK signaling plays an important role in the induction of excessive autophagy after the accumulation of unfolded proteins in Krill-deficient HSPCs, we treated krill ${ }^{\text {cas } 002}$ mutant embryos with PERK-specific inhibitor GSK2656157. GSK2656157 treatment indeed successfully restored the $c m y b$ expression in krill ${ }^{\text {cas } 002}$ embryos
(Figure 6I-6K). Live imaging and immunoblotting analysis further revealed that GSK2656157 treatment inhibited the formation of autophagosomes indicated by LC3-II puncta (Figure 6M-6R and Supplementary information, Figure S18L; $P=0.0091)$. In addition, we performed microinjection of perk/eif $2 a k 3 \mathrm{MO}$ in kril cas002 $^{\text {mutants. }}$ Knockdown of PERK reduced the upregulated level of phosphorylated eif2a (Supplementary information, Figure S19A) and LC3-II (Supplementary information, Figure S19B), meanwhile WISH analysis showed a recovery of $c m y b$ expression in krill ${ }^{c a s 002}$ embryos (Figure 6I and $6 \mathrm{~L}$ ). These data together support the notion that PERK activation-mediated excessive autophagy in the HSPCs causes hematopoiesis failure in $\mathrm{krill}^{\text {cas } 002}$.

\section{Discussion}

Through the characterization of a recessive zebrafish mutant krill ${ }^{\text {cas } 002}$ with hematopoietic defects we have uncovered a novel connection between ribosome biogenesis and autophagy in HSPCs. Loss of krill blocks HSPC proliferation in the CHT region and depletes most downstream hematopoietic lineages during definitive hematopoiesis. Due to the dysfunction of SSU complex, in which Krill functions as a critical component [41], the level of $18 \mathrm{~S}$ rRNA is dramatically reduced, although $28 \mathrm{~S}$ rRNA is spared (Figure 2G-2I). The ribosomal biogenesis defects reduce the rate of protein synthesis (Supplementary information, Figure S9A-S9C) and cause misfolded proteins to aggregate (Figure 6A-6G). Accumulation of misfolded proteins and inefficient protein synthesis trigger PERK activation (Figure $6 \mathrm{H}$ ), which subsequently upregulates autophagy [56, 57] (Figure 4A, 4C-4N and Supplementary information, Figure S16). Inhibition of autophagy or PERK signaling using drug treatments (Figure 5, 3-MA and Baf A1 targeting autophagy; Figure 6J-6R, GSK2656157 targeting PERK signaling) or MOs (Figure 5D and 5E, targeting beclin1; Figure 6I and 6L, targeting perk) successfully rescue hematopoiesis defects in $\mathrm{krill}^{\mathrm{cas} 002}$ mutant embryos.

This finding is distinct from the known regulatory role of apoptosis in bone marrow failure or anemia caused by ribosome disorders [15-18]. Apoptotic signals are normal in HSPCs in the CHT of krill ${ }^{\text {cas } 002}$ embryos (Supplementary information, Figure S11). Neither p53 MO, nor caspase 3 inhibitor, nor $p 53$ null allele, rescued the HSPC defects (Supplementary information, Figure S12, S13A-S13D). Results from our biochemical analyses of Lc3-II level and live imaging of Lc3 puncta in HSPCs, however, attribute hematopoiesis defects in krill cas002 embryos to a hyperactivation of autophagy, which can be inhibited by $\mathrm{Bcl} 2$ overexpression that is known to disrupt the formation of VPS34-Beclin1 complex during auto- 


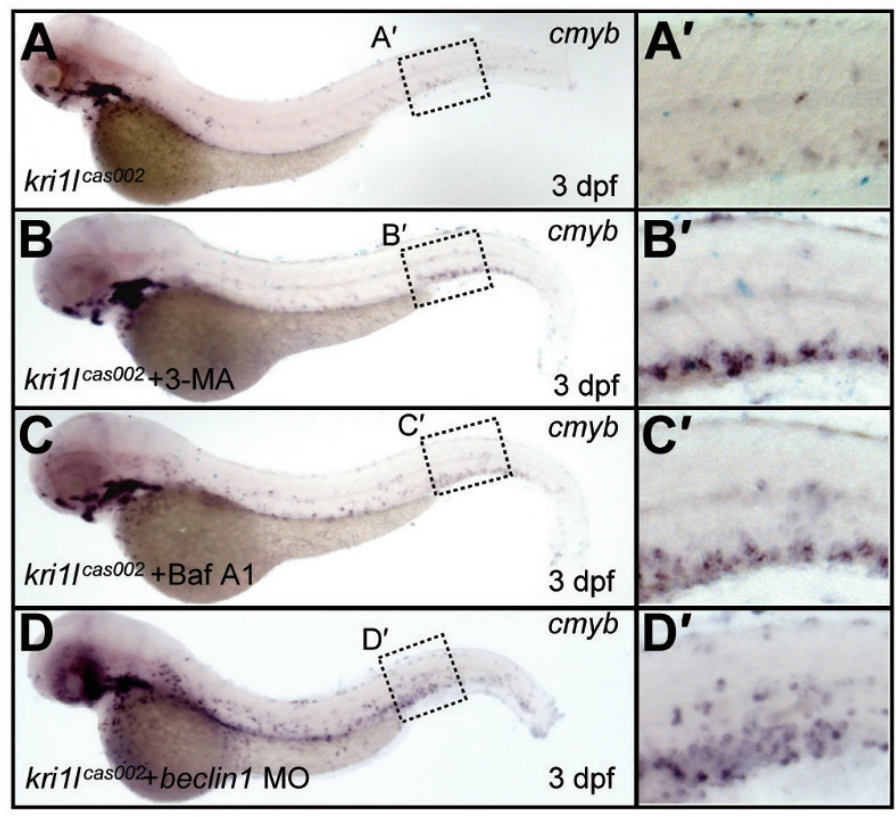

E

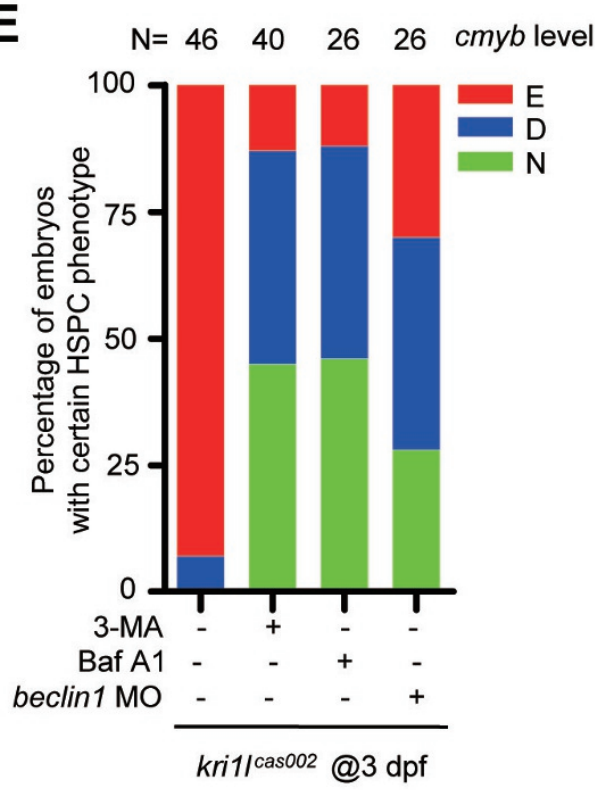

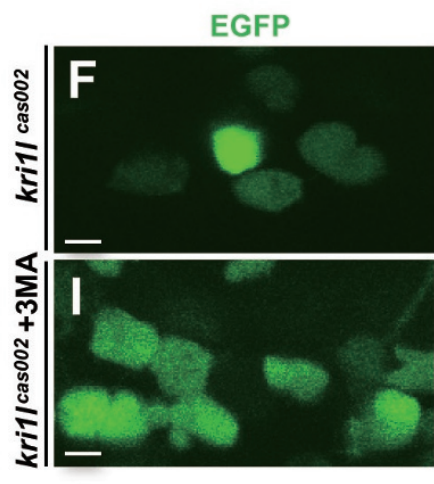
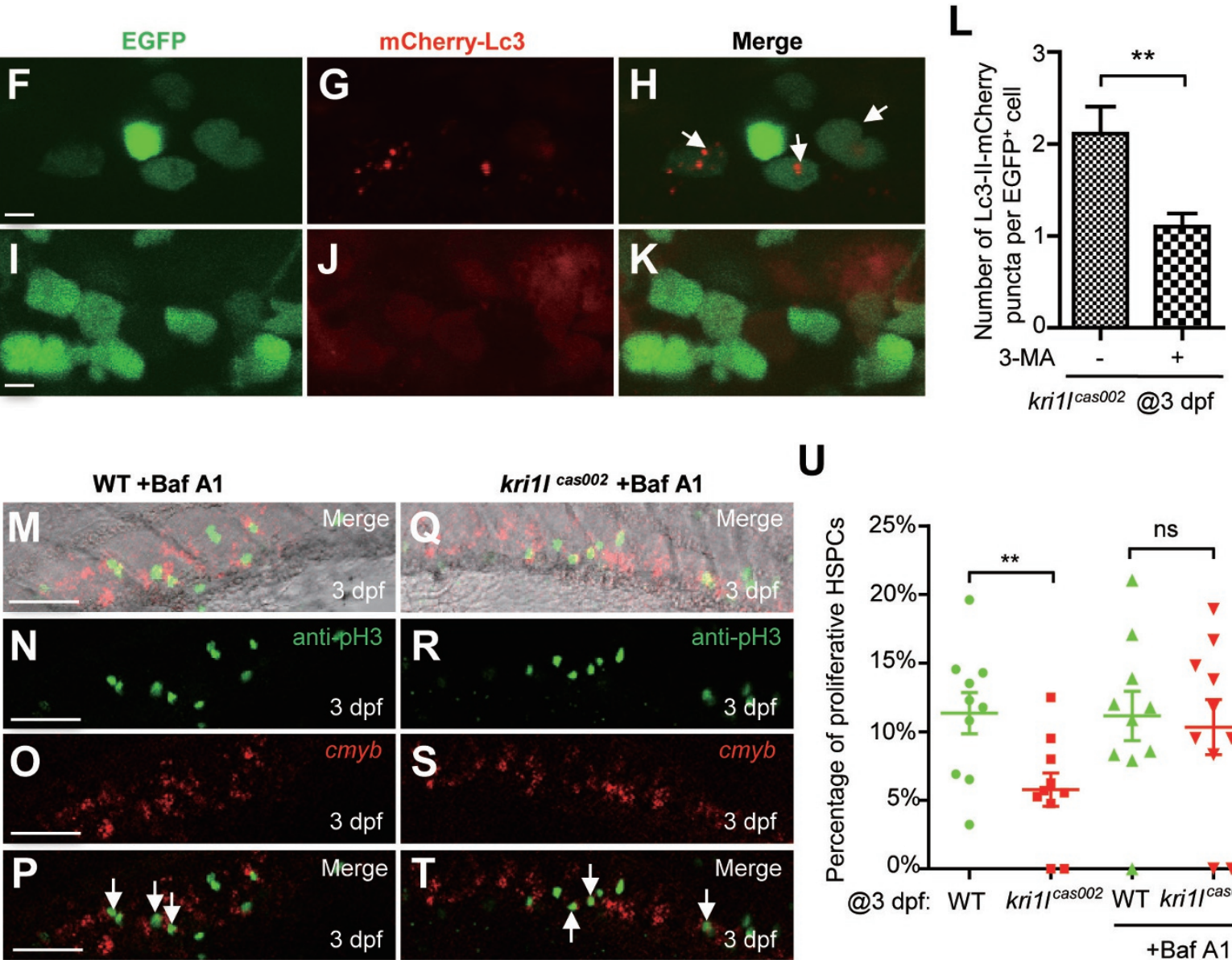

kri1l cas002 +Baf A1
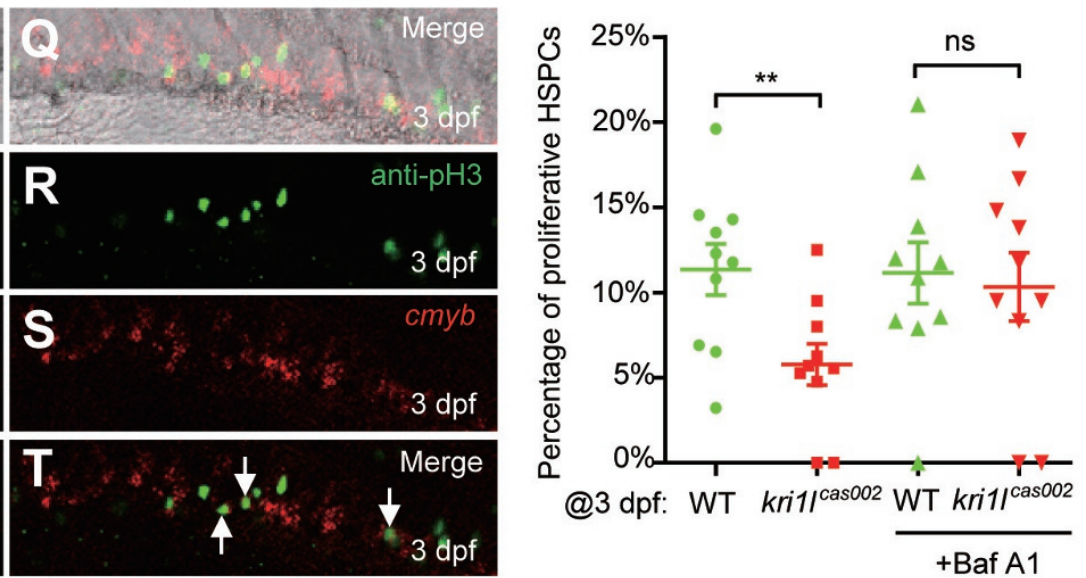
phagy initiation [44] (Figure 4B-4N and Supplementary information, Figure S16). We also tested a small molecular compound Z36, which can mimic Beclin1 to competitively interact with $\mathrm{Bcl} 2$ [62]. Z36 treatment eliminated cmyb expression and increased Lc3-enriched autophagosomes in the CHT region of $\mathrm{Bcl} 2$-overexpressed krill ${ }^{\text {cas } 002}$ embryos (Supplementary information, Figure S18AS18K), further supporting the idea that the hematopoietic phenotype in $k r i l l^{\text {cas } 002}$ is caused by autophagy that is negatively regulated by $\mathrm{Bcl} 2$.

Previous reports have shown that autophagy is essential for HSCs to balance their quiescence, self-renewal and expansion [31]. Activated autophagy driven by FOXO3A-mediated program protects HSCs from starvation-induced apoptosis and maintains HSCs functions [63]. $\operatorname{atg} 7^{-/-}$HSCs show ROS accumulation and a loss of colony formation capacity in replating [32]. Similar observations are obtained in Atg5 knockdown and in FIP2000-null HSCs [64-66]. In contrast, $k$ ill $^{\text {cas } 002}$ HSPCs accumulate excessive autophagosomes that are harmful to HSPC proliferation. Our observation and previous reports together suggest that autophagy functions as a double-edged sword [67] in HSPCs, and an appropriate and fine-tuned autophagy level is important for HSPC proliferation and maintenance.

Morrison group has reported that the rate of protein synthesis in HSCs is highly regulated; either increased or decreased protein synthesis impairs HSC function [68]. Naive HSPCs undergo massive expansion upon arrival in CHT. It is conceivable that HSPCs is more sensitive to ribosome disorders due to the proliferative stress associated with the expansion. Indeed, both autophagy level and proliferation status of HSPCs in AGM are normal before their departure for the CHT (Supplementary information, Figure S10A-S10H and Figure S16A-S16F). In addition, cells in other proliferative tissues, such as cranial region and gut, carry more Lc3-mCherry puncta-positive autophagosomes (data not shown). These observations together suggest proliferative state of stem cells may render them particularly sensitive to Krill deficiency.

How SSU defects cause the accumulation of misfolded proteins remains elusive. Krill deficiency causes defective polysome formation, which includes reduction of functional polysomes or even disassembled polysomes accompanied with excessive $60 \mathrm{~S}$ subunits. Reduced polysomes or excessive $60 \mathrm{~S}$ subunits may lead to disorder in protein synthesis, and cause de novo synthesis of unproductively folded protein. Especially for larger proteins, the well-coupled translation and folding kinetics are vital for the correct production of functional proteins [69]. Unfolded protein response (UPR) was recently reported to regulate HSCs [70]. krill ${ }^{\text {cas } 002}$ HSPCs have a dramatic increase of Hspa4a and ubiquitin-modified proteins, indicating an accumulation of misfolded/unfolded proteins. This leads to the activation of PERK signaling, reflected by an increased level of phospho-eif2a. Importantly, blocking of PERK signaling is sufficient to inhibit excessive autophagy and to rescue definitive hematopoiesis in $k r i l l^{\text {cas } 002}$ embryos. However, only the transcription level of ATF4 (downstream of PERK-eif2a arm of UPR), but not BIP, ATF6, chop, xbpl and xpblls (markers for other signaling arms of UPR), is significantly increased in krill $^{\text {cas } 002}$ mutant (Supplementary information, Figure S20D), implying that either only PERK-eif2a signaling cascade is strongly activated in Krill deficiency-induced UPR, or excessive autophagy in $k$ rill $^{\text {cas } 002}$ mutant is triggered by a PERK-dependent, but UPR-independent signaling mechanism.

A model of S6K-mediated inhibition of insulin pathway has been proposed to explain how RPS19 or RPS7 deficiencies induce the upregulation of autophagy level in peripheral blood cells of patients and zebrafish mor-

Figure 5 Autophagy inhibitors prevent hematopoiesis failure in kri1/ ${ }^{\text {cas } 002}$ mutant embryos. (A-D) Representative images of cmyb expression pattern in kri1/ ${ }^{\text {cas } 002}$ embryos with or without treatment of autophagy inhibitors, or with microinjection of beclin1 MO. kri1/ ${ }^{\text {cas } 002}$ embryos were treated with 3-MA (10 mM) or Baf A1 (25 nM) from 36 hpf to 72 hpf. After cmyb WISH and imaging, all embryos were genotyped; the percentage of the rescue was calculated. 18 out of 40 mutant embryos treated with 3-MA are fully rescued, while 17 out of 40 mutant embryos are rescued partially. 12 out of 26 mutant embryos treated with Baf A1 are fully rescued, while 11 out of 26 mutant embryos are rescued partially. 7 out of 26 mutant embryos injected

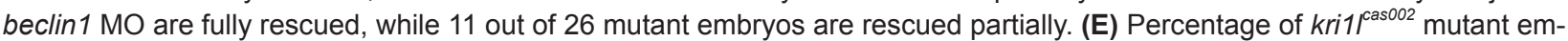
bryos with classified hematopoiesis phenotype with or without treatment of autophagy inhibitors, or with microinjection of beclin1 MO. (F-K) 3-MA inhibits excessive autophagy in HSPCs in kri1/ ${ }^{\text {cas002 }}$ mutant embryos. Representative confocal images of mCherry-Lc3 puncta (autophagosomes) in kri1/ cas002 mutant embryos in Tg (cmyb:egfp) transgenic background are shown. Scale bars, $5 \mu \mathrm{m}$. (L) Quantitative analysis of F-K. The numbers of autophagosomes are decreased in kri1/ as 002 embryos with 3-MA treatment, $p=0.0037$. (M-T) Baf A1 treatment restores HSPCs proliferation in kri1/ ${ }^{\text {cas } 002}$ mutant embryos. Double staining of cmyb RNA (red) and phospho-histone $3(\mathrm{pH} 3)$ protein (green) in the $\mathrm{CHT}$ region of embryos at 3 dpf. $\mathbf{M}$ and $\mathbf{Q}$ show bright-field images overlaid with fluorescent staining. Scale bars, $50 \mu \mathrm{m}$. (U) Percentage of proliferative HSPCs in the CHT of $\mathrm{kri1}^{\mathrm{cas} 002}$ mutant embryos and wild type siblings with or without Baf A1 treatment at $3 \mathrm{dpf} N=10$. Without Baf A1 treatment, $p$ $=0.0014$; with Baf A1 treatment, $P=0.2344$. Error bars represent SEM. ${ }^{* \star} P \leq 0.01 ;{ }^{* *} P \leq 0.001$ (Student's $t$-test). 


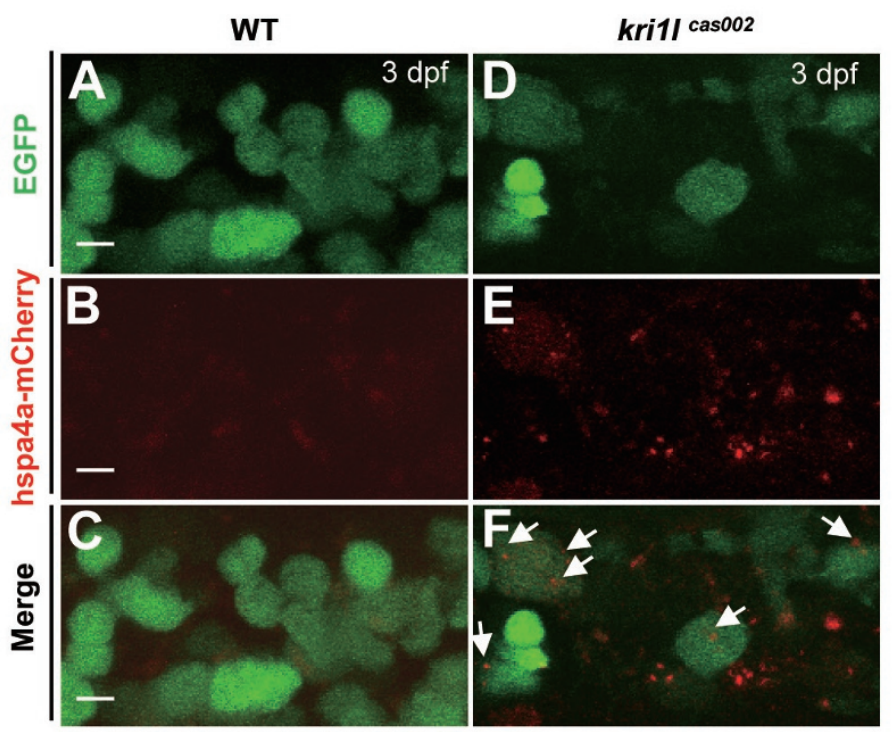

G

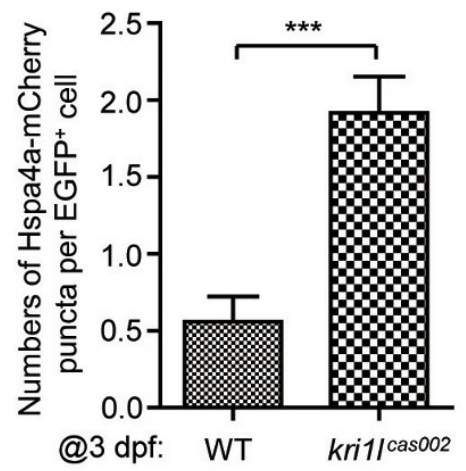

H
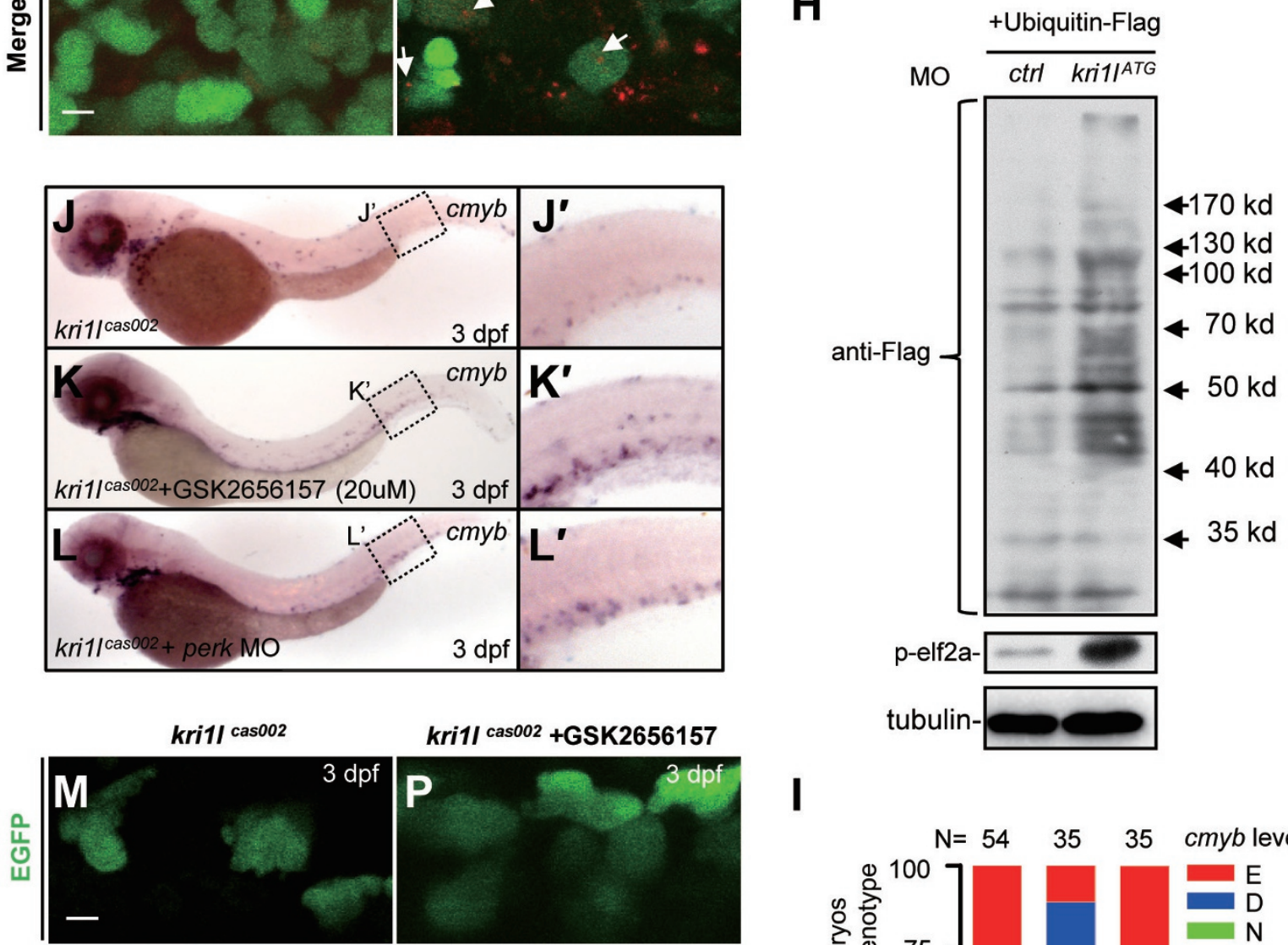

kri1l ${ }^{\text {cas002 +GSK2656157 }}$
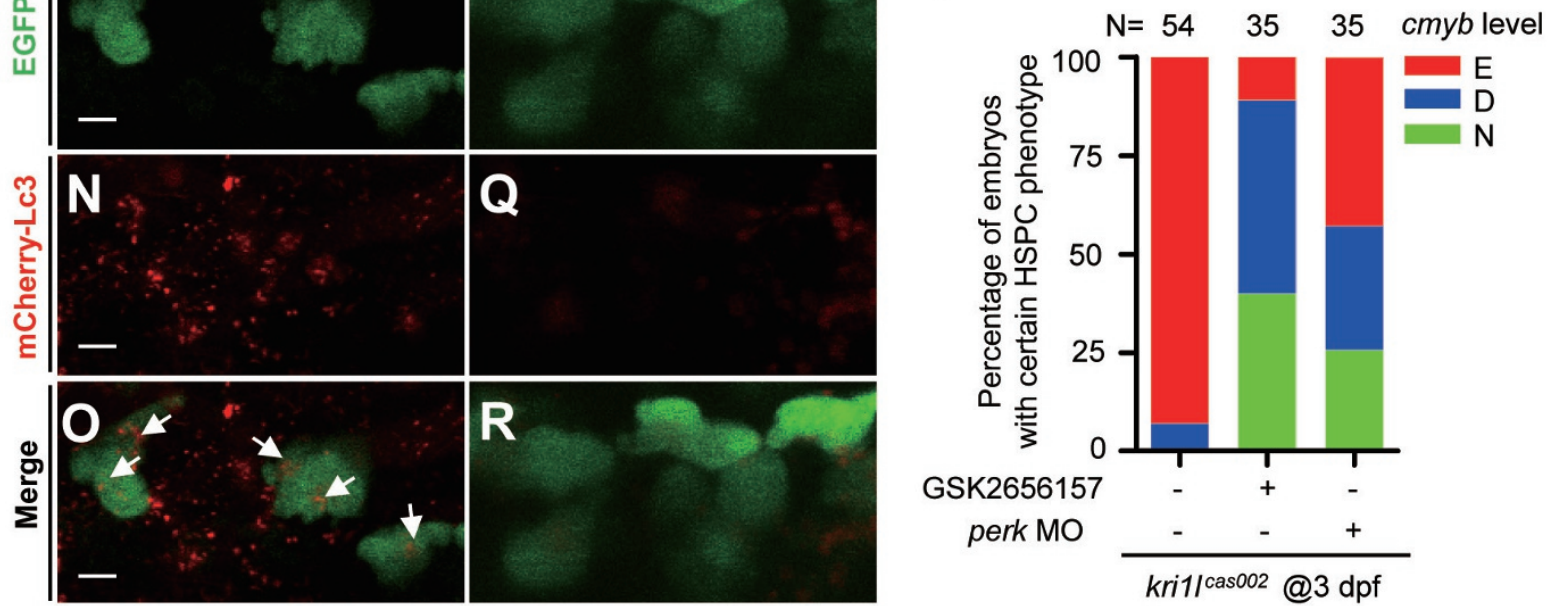
phants [38]. We also show an upregulation of S6K and its downstream effector-RPS6 in krill $l^{\text {cas } 002}$ mutant embryos (Supplementary information, Figure S22A). However, phospho-RPS6 signaling is dominant in peripheral blood cells, but not in $\mathrm{EGFP}^{+}$HSPCs in either wild-type or krill ${ }^{\text {cas } 002}$ mutant (Supplementary information, Figure S22C-S22K), suggesting S6K was not the major regulator to induce excessive autophagy in krill cas002 HSPCs. Taken together, we propose that the deficiency of Krill causes an impaired ribosome biogenesis, resulting in the accumulation of misfolded proteins. Aggregation of misfolded proteins activates PERK signaling, which in turn triggers a significant increase of autophagy. Excessive autophagy finally leads to an inhibition of definitive hematopoiesis. The S6K-RPS6 axis might be involved in the regulation of Krill-deficient peripheral blood cells rather than HSPCs (Supplementary information, Figure S22B). In addition, we found that rapamycin treatment could not rescue hematopoietic failure phenotype in krill mutant (Supplementary information, Figure S20AS20C).

Although $1 \mathrm{mo} 2$ promoter-induced expression of wildtype Krill rescued defective hematopoiesis in krill cas002 embryos (Supplementary information, Figure S21), suggesting a cell autonomous role of Krill in HSPCs, it is also worth to note the presence of other cell types in the CHT. These include niche cells forming the hematopoietic microenvironment and differentiated hematopoietic lineage cells. They may also undergo an upregulation of autophagic flux in $k r i 1 l^{\text {cas } 002}$ mutant. Since appropriate cell-cell interactions in hematopoietic niche are vital for HSPC self-renewal, non-cell autonomous effects may also contribute to the defective hematopoiesis in kril cas $002^{2}$ mutant.

In conclusion, an intact ribosome biogenesis, optimal protein synthesis and an appropriate level of autophagy are critical for HSPC maintenance and proliferation. During the first wave of HSPC expansion in the CHT region, a higher level of protein synthesis is needed, which makes HSPCs more sensitive to dysfunctional ribosome biogenesis [68, 71]. In krill ${ }^{\text {cas } 002}$ HSPCs, impaired ribosome biogenesis causes ribosomal stress and an upregulation of autophagy, which subsequently results in impaired proliferation of HSPCs. Decreased HSPCs can be restored by the treatment of autophagy inhibitors, PERK inhibitor and PERK/Beclin1 knockdown (Figure 7). This study suggests that autophagy level may be useful for the clinical diagnosis of anemia or bone marrow failure caused by ribosomopathies. Our finding also suggests patients with certain types of ribosomopathy may benefit from treatments with autophagy inhibitors, such as lys 05 or spautin-1 [71]. In addition, the mechanism revealed in this study may also play an important role in the progression of other human diseases caused by ribosome abnormalities.

\section{Materials and Methods}

\section{Zebrafish husbandry and MO/mRNA microinjection}

Zebrafish stock maintenance, ENU mutagenesis and positional cloning were performed as previously described $[39,72,73]$. Zebrafish facility and study were approved by Institutional Animal Use Review Board of Institute of Health Sciences, Shanghai Institutes of Biological Sciences, Chinese Academy of Sciences. Morpholino oligonucleotides (MOs) were ordered from Gene Tools, LLC. Capped mRNAs were transcribed from linearized $\mathrm{pCS} 2^{+}$ plasmids (mMessage Machine; Ambion), purified and diluted to $150 \mathrm{ng} / \mu \mathrm{l}$ for microinjection into zebrafish embryos at 1-cell stage.

\section{ENU mutagenesis and positional cloning}

ENU mutagenesis and positional cloning were performed as previously described $[39,72]$. The cas002 (Tu background) allele

Figure 6 Accumulation of unfolded proteins and PERK activation leads to excessive autophagy and hematopoiesis failure. (A-G) Representative confocal images (A-F) and Quantitation (G) of Hspa4a-mCherry puncta (aggregation of misfolded protein) in HSPCs of live zebrafish embryos. Wild-type siblings or kri1/ cas002 mutant embryos in the Tg (cmyb:egfp) transgenic background were injected with hspa4a-mcherry mRNA at one-cell stage and imaged at 3 dpf. The numbers of misfolded protein puncta are increased in kri1/ $/^{\text {cas } 002}$ embryos compared to that in wild-type siblings, $P=0.0002$. Hspa4a-mCherry puncta were counted in over 30 EGFP $^{+}$cells (in the Tg (cmyb: egfp) background) per embryo, total 10-12 embryos. Error bars represent SEM. ${ }^{* *} P \leq 0.01 ;{ }^{* * *} P \leq 0.001$ (Student's $t$-test). Scale bars, $5 \mu \mathrm{m}$. (H) Representative immunoblotting analysis of the level of protein ubiquitination and phospho-eif2a in whole embryo lysates of WT and kri1/cas002 embryos at 3 dpf injected with Flag-tagged ubiquitin plasmid at one-cell stage. Tubulin serves as the loading control. (I) Treatment with PERK inhibitor GSK2656157 or injection of perk MO rescues cmyb expression in HSPCs. Kri1/ ${ }^{\text {cas } 002}$ mutant embryos were treated with GSK2656157 $(20 \mu \mathrm{M})$ from $36 \mathrm{hpf}$ to $72 \mathrm{hpf}$, or injected with perk MO at one-cell stage. After WISH and imaging, all embryos were genotyped. 14 out of 35 mutant embryos treated with GSK2656157 are fully rescued, while 17 out of 35 mutant embryos are rescued partially. 9 out of 35 mutant embryos injected with perk MO are fully rescued, while 11 out of 35 mutant embryos are rescued partially. (J-L) Proportion of kri1/ cas 002 mutant embryos with classified hematopoiesis phenotype with or without GSK2656157 treatment, or with microinjection of perk MO. (M-R) GSK2656157 inhibits excessive autophagy in kri1/cas002 HSPCs. Representative confocal images of mCherry-Lc3 puncta (autophagosomes) in kri1/ ${ }^{\text {cas } 002}$ mutant embryos in Tg (cmyb:egfp) transgenic background are shown. Scale bars, $5 \mu \mathrm{m}$. 
A

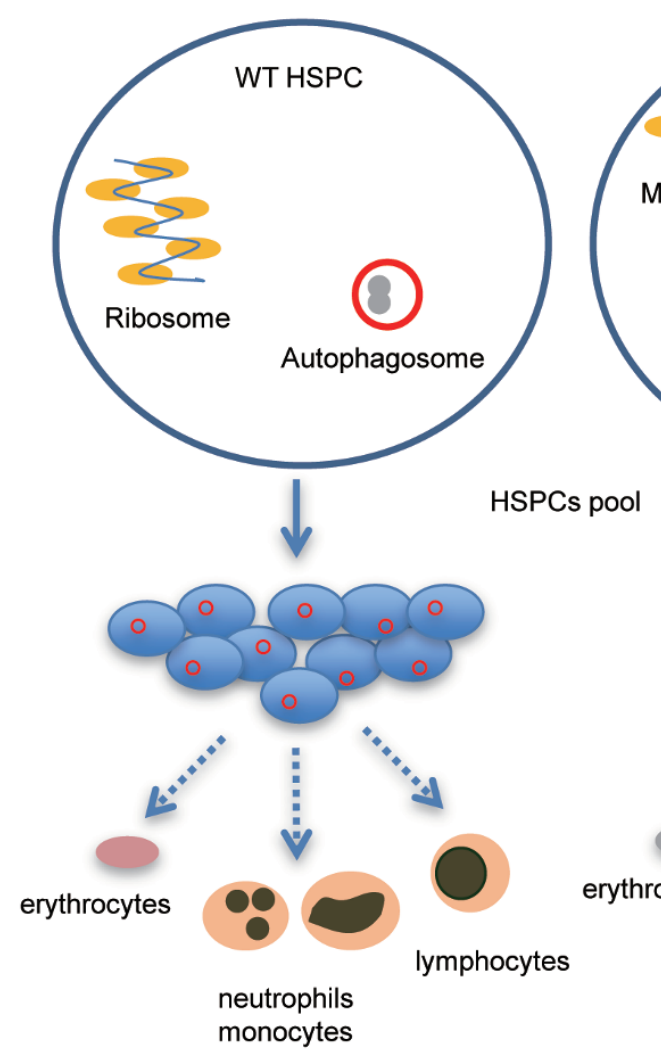

B

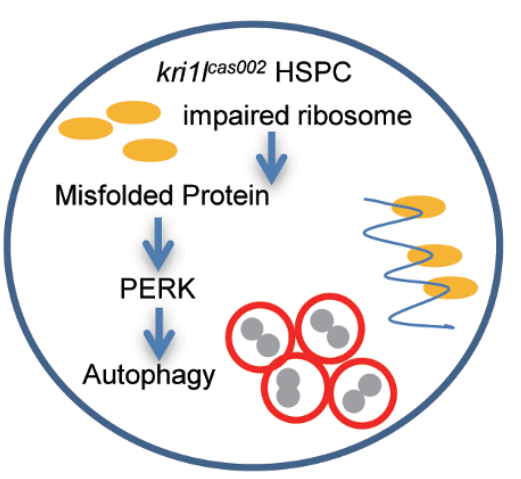

C

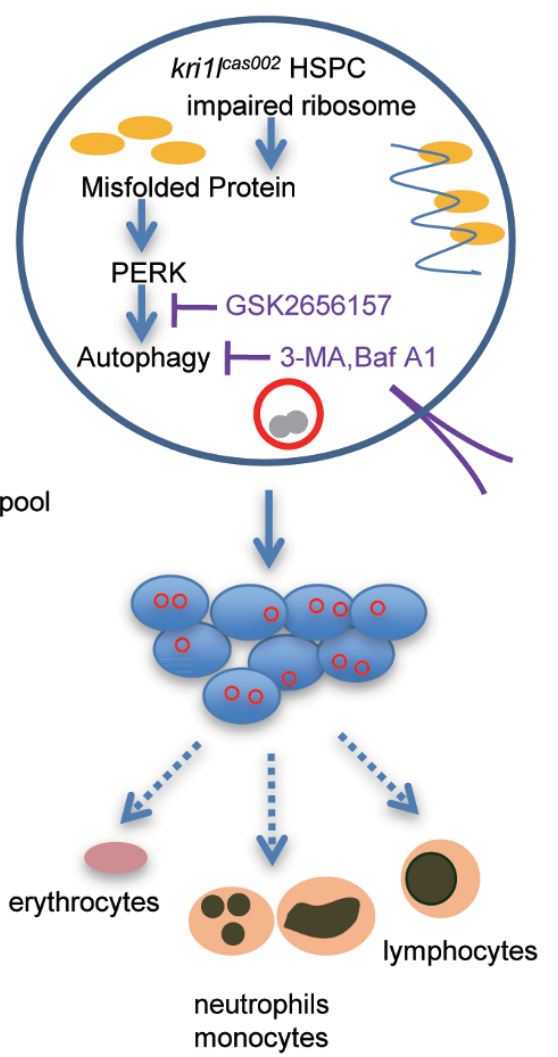

Figure 7 Working model for Kri1l dysfunction-induced hematopoietic defects and its treatment. (A) In normal HSPCs, ribosome biogenesis is tightly regulated; autophagy occurs at an appropriate level. HSPCs expand massively in the CHT region and differentiate into multiple hematopoietic lineages, including erythrocytes, myeloid cells (neutrophils and monocytes) and

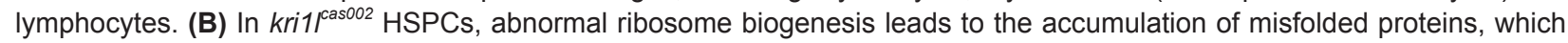
triggers PERK activation and excessive autophagy. The excessive autophagy leads to inhibition of HPSC proliferation, depletion of HSPC pool, and a profound hematopoiesis failure. (C) Blocking of PERK signaling with specific inhibitor (GSK2656157), $\mathrm{Bcl} 2$ overexpression or treatment with autophagy inhibitors (3-MA, Baf A1) reduces autophagy level and restores HSPC proliferation defects and hematopoietic lineage differentiation in kri1/ asoo2 mutant embryo.

was mapped by out-crossing Tu background heterozygous fish into polymorphic WIK background wild-type strain. We scanned the genome for linked SSLP markers by BSA. SSLP markers [74, 75] used for BSA were selected from the Massachusetts General Hospital Zebrafish Server website (http://zebrafish.mgh.harvard. edu). Fine mapping using mainly SSLP markers was carried out to narrow down the genetic interval. The cDNAs of candidate genes in the interval were cloned and sequenced from pooled mutants, and candidate mutation was confirmed by sequencing genomic DNA of individual mutant embryo. All primers used for this study are provided in Supplementary Table 1.

\section{WISH, TUNEL assay and immunostaining}

Antisense RNA probes were transcribed using linearized constructs with T3 or T7 polymerase (Ambion) in the presence of digoxigenin (DIG, Roche)-labeled UTP using the DIG-RNA Labeling Kit (Roche). WISH was performed as described previously using NBT/BCIP (Sigma) as substrates [76, 77]. TUNEL was performed with In Situ Cell Death Detection Kit and TMR
Red Kit (Roche) following manufacturer's instruction. To detect both $c m y b$ RNA and mitosis marker pH3 simultaneously, embryos were first hybridized with the DIG-labeled antisense $c m y b$ RNA probe, incubated at $4{ }^{\circ} \mathrm{C}$ overnight with a peroxidase-conjugated anti-DIG antibody (1:500; Roche), and stained with Alexa Fluor cy3-conjugated tyramide as substrate (PerkinElmer). The embryos were then incubated with primary anti-pH3 (ser10) antibody (1:500; Santa Cruz), and finally incubated with Alexa Fluor 488-labeled goat anti-rabbit IgG antibody (1:500; Invitrogen).

\section{Plasmid construction}

The zebrafish cDNA of krill gene was amplified from reverse transcription products and cloned into $\mathrm{pCS} 2^{+}$vector. For construction of the krill (1-60)-gfp reporter plasmid, the zebrafish cDNA (1-60 aa) of krill gene was amplified and cloned into pCS2 $2^{+}-e g f p$ vector. For construction of $\mathrm{Tg}$ (lmo2:krill) transient transgenesis plasmid, the zebrafish $1 m o 2$ promoter was obtained by PCR amplification from Imo2-Cre-PBSK-I-SceI plasmid, and then cloned into the Tol 2 transposon backbone together with full-length krill gene 
[78]. For construction of the mcherry-lc3 plasmid, the PCR was performed to generate mcherry cDNA without the termination codon, and then replace the EGFP coding region of $\mathrm{pEGFP-C1}$, and the resulting plasmid was named mCherry-C1. The fragment of $l c 3$ was inserted into the corresponding sites in the mCherry- $\mathrm{C} 1$ plasmid. The zebrafish cDNA of hspa4a gene without the termination codon was amplified and cloned into $\mathrm{pCS} 2^{+}$vector. Then mcherry cDNA was generated by PCR and inserted into $\mathrm{pCS} 2^{+}-h s p a 4 a$.

Live imaging of autophagy and unfolded protein in HSPCs

$m$ Cherry-Lc3 mRNA or hspa4a-mCherry mRNA was transcribed via the mMessage mMachine SP6 kit (Ambion), and then injected into $\operatorname{Tg}(c m y b: e g f p)$ transgenic zebrafish embryos at onecell stage. The live embryos were anesthetized with tricaine and mounted in 3\% low melting point agarose for imaging with Olympus FV1000 scanning confocal microscope (under a 60×/1.00 NA water-immersion objective) [79].

\section{Immunoblotting analysis}

Embryos were deyolked [80], and then homogenized in lysis buffer (20 mM Tris- $\mathrm{HCl}$ (pH 7.4), $150 \mathrm{mM} \mathrm{NaCl,} 5$ mM EDTA, $10 \%$ glycerol, $0.1 \%$ Triton X-100, protease inhibitor cocktail and phosphatase inhibitor (Roche)). Protein lysates were separated on SDS-PAGE, transferred to nitrocellulose membrane (Amersham Biosciences), and incubated with anti-LC3 (Cell Signaling Technology, 1:1 000), phos-S6K (Cell Signaling Technology, 1:1 000), phos-RPS6 (Cell Signaling Technology, 1:1 000), phos-elf2a (Cell Signaling Technology, 1:1 000), p62 (MBL,1:1 000), Beclin1 (abcam, 1:5 000), p53 (a gift from Jinrong Peng, Zhejiang University) or anti- $\alpha$-tubulin (Sigma, 1:2 000) antibodies, and then HRP-conjugated secondary Antibodies.

\section{Small molecular compound treatment}

Final concentrations of chemicals used in this study were 5 $\mu \mathrm{M}$ chloroquine (Fluka Sigma-Aldrich), $10 \mathrm{mM}$ 3-MA (Sigma), $25 \mathrm{nM}$ Baf A1 (Santa Cruz), $10 \mu \mathrm{M}$ Z36 (Sigma) and $20 \mu \mathrm{M}$ GSK2656157 (Selleck). Embryos were incubated with small molecular chemicals in embryo medium at $28.5^{\circ} \mathrm{C}$ until collection.

\section{Transmission electron microscopy}

For electron microscopy analysis, $3 \mathrm{dpf}$ zebrafish embryos were fixed in a mixture of $2 \%$ paraformaldehyde and $0.2 \%$ glutaraldehyde in $0.1 \mathrm{M}$ phosphate buffer. The embryos were washed in PBS-glycine to quench free aldehydes, then embedded in gelatin and infiltrated in $2.3 \mathrm{M}$ sucrose, and subjected to rapid freezing in liquid $\mathrm{N}_{2} .50 \mathrm{nM}$ thick cryosections were cut at $-120{ }^{\circ} \mathrm{C}$ using an Ultracut-S ultra microtome (Leica Microsystems). Sections were directly viewed in a FEI Tecnai G2 Spirit Twin electron microscope (FEI).

\section{Quantitative PCR analysis}

Total RNAs were extracted from 20 zebrafish embryos using Trizol reagent (Invitrogen). RNA was reverse-transcribed using random hexamers and SuperScript III Reverse Transcriptase (Invitrogen). $2 \times$ PCR Mix (TaKaRa, Premix Ex Taq) containing SYBR Green I was used for the real-time quantitative PCR analysis with the Applied Biosystems 7900HT Fast Real-Time PCR System. The relative expression values were normalized against the internal control actin (QPCR primer sequences were listed in Supplemen- tary information, Table S1).

Quantitation of rRNA and polysome level

As previously described [21], total RNA was extracted from WT or krill $l^{\text {cas } 002}$ mutant zebrafish and then analyzed on an Agilent 2100 E-Bioanalyser according to the manufacturer's instructions. $140 \mathrm{WT}$ or krill $l^{\text {cas } 002}$ larvae at $72 \mathrm{hpf}$ were deyolked, washed and resuspended in cold lysis buffer (50 mM Tris- $\mathrm{HCl}(\mathrm{pH} 7.4), 150$ $\mathrm{mM} \mathrm{KCl}, 10 \mathrm{mM} \mathrm{MgCL2}, 1 \%$ Triton X-100, 2 mM DTT, 0.5\% sodium deoxycholate and $0.1 \mathrm{mg} / \mathrm{ml}$ cycloheximide) containing $100 \mathrm{U} / \mathrm{ml}$ RNase inhibitor (Promega), Complete Protease Inhibitor Cocktail (Roche), sodium vanadate, sodium fluoride and PMSF. Next, samples were homogenized by small pestles. Lysates were incubated on ice for $15 \mathrm{~min}$ and centrifuged (12 $000 \mathrm{rpm}, 10 \mathrm{~min}$ at $4{ }^{\circ} \mathrm{C}$ ) to pellet the nuclei and cellular debris. The supernatant of each sample was loaded onto a continuous $15 \%-45 \%$ (w/v) sucrose gradient in high salt resolving buffer (20 mM HEPES (pH7.4), $150 \mathrm{mM} \mathrm{KCl}$, and $10 \mathrm{mM} \mathrm{MgCl} 2$ ) generated by a Biocomp gradient master. The mixture was next centrifuged in a Beckman SW41ti rotor (Beckman Coulter) at $36000 \mathrm{rpm}$ for $3.5 \mathrm{~h}$ at $4{ }^{\circ} \mathrm{C}$, and the absorbance at $254 \mathrm{~nm}$ was determined with an EM-1 UV Monitor (Bio-Rad).

\section{Acknowledgment}

We thank Xiaolan Peng, Ting-Xi Liu, Jinrong Peng, Zhixue Liu, Wei-Li Zhao, Jiang Zhu, Jing-Yi Shi, Qing Chen, Li-Ting Chen, Fan Yang and Zhong Zheng for technical support and suggestions. This work was supported by grants from National Natural Science Foundation of China (31371461 to WJP, 81123005 to State Key Laboratory for Medical Genomics, 31301209 to CBJ, 31301196 to MD), Inner Mongolia Natural Science Foundation (2015BS0801 to XEJ), Science and Technology Commission of Shanghai Municipality (13JC1406400) and National Thousand Talents Program for Distinguished Young Scholars to WJP.

\section{References}

1 Galloway JL, Zon LI. Ontogeny of hematopoiesis: examining the emergence of hematopoietic cells in the vertebrate embryo. Curr Top Dev Biol 2003; 53:139-158.

2 Orkin SH, Zon LI. Hematopoiesis: an evolving paradigm for stem cell biology. Cell 2008; 132:631-644.

3 Morrison SJ, Uchida N, Weissman IL. The biology of hematopoietic stem cells. Annu Rev Cell Dev Biol 1995; 11:35-71.

4 Bertrand JY, Traver D. Hematopoietic cell development in the zebrafish embryo. Curr Opin Hematol 2009; 16:243-248.

5 Paw BH, Zon LI. Zebrafish: a genetic approach in studying hematopoiesis. Curr Opin Hematol 2000; 7:79-84.

6 Song HD, Sun XJ, Deng M, et al. Hematopoietic gene expression profile in zebrafish kidney marrow. Proc Natl Acad Sci USA 2004; 101:16240-16245.

7 Bertrand JY, Kim AD, Teng S, Traver D. CD41+ cmyb+ precursors colonize the zebrafish pronephros by a novel migration route to initiate adult hematopoiesis. Development 2008; 135:1853-1862.

8 Jin H, Xu J, Wen Z. Migratory path of definitive hematopoietic stem/progenitor cells during zebrafish development. Blood 2007; 109:5208-5214. 
9 Kissa K, Murayama E, Zapata A, et al. Live imaging of emerging hematopoietic stem cells and early thymus colonization. Blood 2008; 111:1147-1156.

10 Murayama E, Kissa K, Zapata A, et al. Tracing hematopoietic precursor migration to successive hematopoietic organs during zebrafish development. Immunity 2006; 25:963-975.

11 Sondalle SB, Baserga SJ. Human diseases of the SSU processome. Biochim Biophys Acta 2014; 1842:758-764.

12 Liu JM, Ellis SR. Ribosomes and marrow failure: coincidental association or molecular paradigm? Blood 2006; 107:45834588.

13 Narla A, Ebert BL. Ribosomopathies: human disorders of ribosome dysfunction. Blood 2010; 115:3196-3205.

14 Aguissa-Toure AH, Da Costa L, Leblanc T, Tchernia G, Fribourg S, Gleizes PE. Diamond-Blackfan anemia reveals the dark side of ribosome biogenesis. Med Sci (Paris) 2009; 25:69-76.

15 Taylor AM, Humphries JM, White RM, Murphey RD, Burns CE, Zon LI. Hematopoietic defects in rps29 mutant zebrafish depend upon p53 activation. Exp Hematol 2012; 40:228-237.

16 Barlow JL, Drynan LF, Hewett DR, et al. A p53-dependent mechanism underlies macrocytic anemia in a mouse model of human 5q- syndrome. Nat Med 2010; 16:59-66.

17 McGowan KA, Li JZ, Park CY, et al. Ribosomal mutations cause p53-mediated dark skin and pleiotropic effects. Nat Genet 2008; 40:963-970.

18 Danilova N, Sakamoto KM, Lin S. Ribosomal protein S19 deficiency in zebrafish leads to developmental abnormalities and defective erythropoiesis through activation of $\mathrm{p} 53$ protein family. Blood 2008; 112:5228-5237.

19 Chakraborty A, Uechi T, Higa S, Torihara H, Kenmochi N. Loss of ribosomal protein L11 affects zebrafish embryonic development through a p53-dependent apoptotic response. PLoS One 2009; 4:e4152.

20 Provost E, Wehner KA, Zhong X, et al. Ribosomal biogenesis genes play an essential and p53-independent role in zebrafish pancreas development. Development 2012; 139:3232-3241.

21 Boglev Y, Badrock AP, Trotter AJ, et al. Autophagy induction is a Tor- and Tp53-independent cell survival response in a zebrafish model of disrupted ribosome biogenesis. PLoS Genet 2013; 9:e1003279.

22 Torihara H, Uechi T, Chakraborty A, Shinya M, Sakai N, Kenmochi N. Erythropoiesis failure due to RPS19 deficiency is independent of an activated Tp53 response in a zebrafish model of Diamond-Blackfan anaemia. Br J Haematol 2011; 152:648-654.

23 Dutt S, Narla A, Lin K, et al. Haploinsufficiency for ribosomal protein genes causes selective activation of p53 in human erythroid progenitor cells. Blood 2011; 117:2567-2576.

24 Zhang Y, Duc AC, Rao S, et al. Control of hematopoietic stem cell emergence by antagonistic functions of ribosomal protein paralogs. Dev Cell 2013; 24:411-425.

25 Hara T, Nakamura K, Matsui M, et al. Suppression of basal autophagy in neural cells causes neurodegenerative disease in mice. Nature 2006; 441:885-889.

26 Komatsu M, Waguri S, Chiba T, et al. Loss of autophagy in the central nervous system causes neurodegeneration in mice. Nature 2006; 441:880-884.

27 Espina V, Liotta LA. What is the malignant nature of human ductal carcinoma in situ? Nat Rev Cancer 2011; 11:68-75.

28 Degenhardt K, Mathew R, Beaudoin B, et al. Autophagy promotes tumor cell survival and restricts necrosis, inflammation, and tumorigenesis. Cancer Cell 2006; 10:51-64.

29 He C, Klionsky DJ. Regulation mechanisms and signaling pathways of autophagy. Annu Rev Genet 2009; 43:67-93.

30 Funderburk SF, Wang QJ, Yue Z. The Beclin 1-VPS34 complex--at the crossroads of autophagy and beyond. Trends Cell Biol 2010; 20:355-362.

31 Guan JL, Simon AK, Prescott M, et al. Autophagy in stem cells. Autophagy 2013; 9:830-849.

32 Mortensen M, Soilleux EJ, Djordjevic G, et al. The autophagy protein Atg7 is essential for hematopoietic stem cell maintenance. J Exp Med 2011; 208:455-467.

33 Miller BC, Zhao Z, Stephenson LM, et al. The autophagy gene ATG5 plays an essential role in B lymphocyte development. Autophagy 2008; 4:309-314.

34 Pua HH, Dzhagalov I, Chuck M, Mizushima N, He YW. A critical role for the autophagy gene Atg 5 in T cell survival and proliferation. J Exp Med 2007; 204:25-31.

35 Kundu M, Lindsten T, Yang CY, et al. Ulk1 plays a critical role in the autophagic clearance of mitochondria and ribosomes during reticulocyte maturation. Blood 2008; 112:14931502.

36 Mortensen M, Ferguson DJ, Edelmann M, et al. Loss of autophagy in erythroid cells leads to defective removal of mitochondria and severe anemia in vivo. Proc Natl Acad Sci USA 2010; 107:832-837.

37 Sandoval H, Thiagarajan P, Dasgupta SK, et al. Essential role for Nix in autophagic maturation of erythroid cells. Nature 2008; 454:232-235.

38 Heijnen HF, van Wijk R, Pereboom TC, et al. Ribosomal protein mutations induce autophagy through S6 kinase inhibition of the insulin pathway. PLoS Genet 2014; 10:e1004371.

39 Bahary N, Davidson A, Ransom D, et al. The Zon laboratory guide to positional cloning in zebrafish. Methods Cell Biol 2004; 77:305-329.

40 Whitehead GG, Makino S, Lien CL, Keating MT. fgf20 is essential for initiating zebrafish fin regeneration. Science 2005; 310:1957-1960.

41 Sasaki T, Toh EA, Kikuchi Y. Yeast Krrlp physically and functionally interacts with a novel essential Krilp, and both proteins are required for $40 \mathrm{~S}$ ribosome biogenesis in the nucleolus. Mol Cell Biol 2000; 20:7971-7979.

42 Yuan H, Zhou J, Deng M, et al. Small ubiquitin-related modifier paralogs are indispensable but functionally redundant during early development of zebrafish. Cell Res 2010; 20:185196.

43 Chen J, Ng SM, Chang C, et al. p53 isoform delta113p53 is a p53 target gene that antagonizes p53 apoptotic activity via BclxL activation in zebrafish. Genes Dev 2009; 23:278-290.

44 Pattingre S, Tassa A, Qu X, et al. Bcl-2 antiapoptotic proteins inhibit Beclin 1-dependent autophagy. Cell 2005; 122:927939.

45 Maiuri MC, Le Toumelin G, Criollo A, et al. Functional and physical interaction between $\mathrm{Bcl}-\mathrm{X}(\mathrm{L})$ and a $\mathrm{BH} 3$-like domain in Beclin-1. EMBO J 2007; 26:2527-2539.

46 Klionsky DJ, Abdalla FC, Abeliovich H, et al. Guidelines for the use and interpretation of assays for monitoring autophagy. 
Autophagy 2012; 8:445-544.

47 Klionsky DJ, Abeliovich H, Agostinis P, et al. Guidelines for the use and interpretation of assays for monitoring autophagy in higher eukaryotes. Autophagy 2008; 4:151-175.

48 Berghmans S, Murphey RD, Wienholds E, et al. tp53 mutant zebrafish develop malignant peripheral nerve sheath tumors. Proc Natl Acad Sci USA 2005; 102:407-412.

49 Galavotti S, Bartesaghi S, Faccenda D, et al. The autophagy-associated factors DRAM1 and p62 regulate cell migration and invasion in glioblastoma stem cells. Oncogene 2013; 32:699-712.

50 He C, Klionsky DJ. Analyzing autophagy in zebrafish. Autophagy 2010; 6:642-644.

51 He C, Bartholomew CR, Zhou W, Klionsky DJ. Assaying autophagic activity in transgenic GFP-Lc3 and GFP-Gabarap zebrafish embryos. Autophagy 2009; 5:520-526.

52 Kimmelman AC. The dynamic nature of autophagy in cancer. Genes Dev 2011; 25:1999-2010.

53 Schonewolf CA, Mehta M, Schiff D, et al. Autophagy inhibition by chloroquine sensitizes HT-29 colorectal cancer cells to concurrent chemoradiation. World J Gastrointest Oncol 2014; 6:74-82.

54 Wu YT, Tan HL, Shui G, et al. Dual role of 3-methyladenine in modulation of autophagy via different temporal patterns of inhibition on class I and III phosphoinositide 3-kinase. J Biol Chem 2010; 285:10850-10861.

55 Yamamoto A, Tagawa Y, Yoshimori T, Moriyama Y, Masaki R, Tashiro Y. Bafilomycin A1 prevents maturation of autophagic vacuoles by inhibiting fusion between autophagosomes and lysosomes in rat hepatoma cell line, H-4-II-E cells. Cell Struct Funct 1998; 23:33-42.

56 Hoyer-Hansen M, Jaattela M. Connecting endoplasmic reticulum stress to autophagy by unfolded protein response and calcium. Cell Death Differ 2007; 14:1576-1582.

57 Kouroku Y, Fujita E, Tanida I, et al. ER stress (PERK/eIF2alpha phosphorylation) mediates the polyglutamine-induced LC3 conversion, an essential step for autophagy formation. Cell Death Differ 2007; 14:230-239.

58 Buchberger A, Bukau B, Sommer T. Protein quality control in the cytosol and the endoplasmic reticulum: brothers in arms. Mol Cell 2010; 40:238-252.

59 Crawford KC, Vega Flores M, Oehlers SH, Hall CJ, Crosier $\mathrm{KE}$, Crosier PS. Zebrafish heat shock protein a4 genes in the intestinal epithelium are up-regulated during inflammation. Genesis 2011; 49:905-911.

60 Amm I, Sommer T, Wolf DH. Protein quality control and elimination of protein waste: the role of the ubiquitin-proteasome system. Biochim Biophys Acta 2014; 1843:182-196.

61 Wek RC, Cavener DR. Translational control and the unfolded protein response. Antioxid Redox Signal 2007; 9:2357-2371.

62 Lin J, Zheng Z, Li Y, et al. A novel Bcl-XL inhibitor Z36 that induces autophagic cell death in Hela cells. Autophagy 2009; 5:314-320.

63 Warr MR, Binnewies M, Flach J, et al. FOXO3A directs a protective autophagy program in haematopoietic stem cells. Nature 2013; 494:323-327.

64 Salemi S, Yousefi S, Constantinescu MA, Fey MF, Simon HU. Autophagy is required for self-renewal and differentia- tion of adult human stem cells. Cell Res 2012; 22:432-435.

65 Liu F, Guan JL. FIP200, an essential component of mammalian autophagy is indispensible for fetal hematopoiesis. Autophagy 2011; 7:229-230.

66 Liu F, Lee JY, Wei H, et al. FIP200 is required for the cell-autonomous maintenance of fetal hematopoietic stem cells. Blood 2010; 116:4806-4814.

67 Shintani T, Klionsky DJ. Autophagy in health and disease: a double-edged sword. Science 2004; 306:990-995.

68 Signer RA, Magee JA, Salic A, Morrison SJ. Haematopoietic stem cells require a highly regulated protein synthesis rate. Nature 2014; 509:49-54.

69 Kim YE, Hipp MS, Bracher A, Hayer-Hartl M, Hartl FU. Molecular chaperone functions in protein folding and proteostasis. Annu Rev Biochem 2013; 82:323-355.

70 van Galen P, Kreso A, Mbong N, et al. The unfolded protein response governs integrity of the haematopoietic stem-cell pool during stress. Nature 2014; 510:268-272.

71 McAfee Q, Zhang Z, Samanta A, et al. Autophagy inhibitor Lys05 has single-agent antitumor activity and reproduces the phenotype of a genetic autophagy deficiency. Proc Natl Acad Sci USA 2012; 109:8253-8258.

72 Mullins MC, Nusslein-Volhard C. Mutational approaches to studying embryonic pattern formation in the zebrafish. Curr Opin Genet Dev 1993; 3:648-654.

73 Kimmel CB, Ballard WW, Kimmel SR, Ullmann B, Schilling TF. Stages of embryonic development of the zebrafish. Dev Dyn 1995; 203:253-310.

74 Knapik EW, Goodman A, Ekker M, et al. A microsatellite genetic linkage map for zebrafish (Danio rerio). Nat Genet 1998; 18:338-343.

75 Shimoda N, Knapik EW, Ziniti J, et al. Zebrafish genetic map with 2000 microsatellite markers. Genomics 1999; 58:219232.

76 Thisse C, Thisse B. High-resolution in situ hybridization to whole-mount zebrafish embryos. Nat Protoc 2008; 3:59-69.

77 Zhang Y, Bai XT, Zhu KY, et al. In vivo interstitial migration of primitive macrophages mediated by JNK-matrix metalloproteinase 13 signaling in response to acute injury. J Immunol 2008; 181:2155-2164.

78 Wang L, Zhang Y, Zhou T, et al. Functional characterization of Lmo2-Cre transgenic zebrafish. Dev Dyn 2008; 237:21392146.

79 Le Guyader D, Redd MJ, Colucci-Guyon E, et al. Origins and unconventional behavior of neutrophils in developing zebrafish. Blood 2008; 111:132-141.

80 Link V, Shevchenko A, Heisenberg CP. Proteomics of early zebrafish embryos. BMC Dev Biol 2006; 6:1.

(Supplementary information is linked to the online version of the paper on the Cell Research website.)

(c) (i)(-) (2) This work is licensed under the Creative Commons Attribution-NonCommercial-No Derivative Works 3.0 Unported License. To view a copy of this license, visit http:// creativecommons.org/licenses/by-nc-nd/3.0 